\title{
Mutation and Microsatellite Burden Predict Response to PD-1 Inhibition in Children with Germline DNA Replication Repair Deficiency
}

Uri Tabori ( $\square$ uri.tabori@sickkids.ca )

Hospital for Sick Children

Daniel Morgenstern

Division of Haematology Oncology, The Hospital for Sick Children, Toronto, Canada https://orcid.org/0000-0002-4859-1108

\section{Sumedha Sudhaman}

Program in Genetics and Genome Biology, The Hospital for Sick Children, Toronto, Canada

\section{Anirban Das}

The Hospital for Sick Children https://orcid.org/0000-0001-7653-9529

\section{Ailish Coblentz}

Department of Diagnostic Imaging, The Hospital for Sick Children, Toronto, Canada

Jiil Chung

Program in Genetics and Genome Biology, The Hospital for Sick Children, Toronto, Canada

\section{Simone Stone}

Princess Margaret Cancer Centre, University Health Network, Toronto, Canada

\section{Noor Alsafwani}

Division of Neuropathology, Department of Laboratory Medicine and Pathobiology, University of Toronto, Canada https://orcid.org/0000-0002-9449-9708

\section{Vanja Cabric}

Division of Haematology Oncology, The Hospital for Sick Children, Toronto, Canada

\section{Liana Nobre}

Division of Haematology Oncology, The Hospital for Sick Children, Toronto, Canada

\section{Vanessa Bianchi}

Program in Genetics and Genome Biology, The Hospital for Sick Children, Toronto, Canada

\section{Melissa Edwards}

Program in Genetics and Genome Biology, The Hospital for Sick Children, Toronto, Canada

\section{Lauren Sambira}

Division of Haematology Oncology, The Hospital for Sick Children, Toronto, Canada

\section{Shlomi Constantini}

Tel Aviv University

\section{Rina Dvir}


Department of Pediatric Hematology-Oncology, Tel-Aviv Sourasky Medical Center, Tel-Aviv, Israel

\section{Michal Yalon}

The Chaim Sheba Medical Center

\section{Gadi Campino}

Department of Pediatric Hematology-Oncology, Sheba Medical Center, Ramat Gan, Israel

\section{Shani Caspi}

Department of Pediatric Hematology-Oncology, Sheba Medical Center, Ramat Gan, Israel

\section{Valerie Larouche}

Centre Hospitalier Universitaire de Quebec-Universite Laval

\section{Alyssa Reddy}

Departments of Neurology and Pediatrics, University of California, San Francisco, California, USA

\section{Michael Osborn}

Women's and Children's Hospital, North Adelaide, Australia

\section{Gary Mason}

Department of Pediatrics, University of Pittsburgh School of Medicine, Pittsburgh, USA

\section{Scott Lindhorst}

Neuro-Oncology, Department of Neurosurgery, and Department of Medicine, Division of Hematology/Medical Oncology, Medical University of South Carolina, Charleston, USA

\section{Annika Bronsema}

Department of Pediatric Hematology and Oncology, University Medical Center Hamburg-Eppendorf, Hamburg, Germany

\section{Vanan Magimairajan}

Department of Pediatric Hematology-Oncology, Cancer Care Manitoba; Research Institute in Oncology and Hematology, University of Manitoba, Winnipeg, Canada

\section{Enrico Opocher}

Pediatric Hematology, Oncology and Stem Cell Transplant Division, Padua University Hospital, Padua, Italy

\section{Rebecca De Mola}

Oregon Health and Science University, Portland, Oregon, USA

\section{Magnus Sabel}

Department of Pediatrics, Institute of Clinical Sciences, Sahlgrenska Academy, University of Gothenburg \& Queen Silvia Children's Hospital, Sahlgrenska Univeristy Hospital, Gothenburg, Sweden https://orcid.org/0000-0002-3072-657X

\section{Charlotta Frojd}

Department of Pediatrics, Institute of Clinical Sciences, Sahlgrenska Academy, University of Gothenburg \& Queen Silvia Children's Hospital, Sahlgrenska Univeristy Hospital, Gothenburg, Sweden

\section{David Sumerauer}

Charles University and University Hospital Motol

\section{David Samuel}


Valley Children's Hospital https://orcid.org/0000-0001-6850-4932

\section{Kristina Cole}

The Children's Hospital of Philadelphia

\section{Stefano Chiaravalli}

Pediatric Unit, Fondazione IRCCS Istituto Nazionale dei Tumori, Milano, Italy

\section{Maura Massimino}

Fondazione IRCCS Istituto Nazionale Tumori

\section{Patrick Tomboc}

Department of Pediatrics, Ruby Memorial Hospital, West Virginia University, West Virginia, USA https://orcid.org/0000-0002-1255-4719

\section{David Ziegler}

Sydney Children's Hospital https://orcid.org/0000-0001-7451-7916

\section{Ben George}

Division of Hematology and Oncology, Department of Medicine, Medical College of Wisconsin, Milwaukee, Milwaukee, Wisconsin, USA

\section{An Van Damme}

Department of Pediatric Hematology and Oncology, Saint Luc University Hospital, Université Catholique de Louvain, Brussels Belgium

\section{Nobuko Hijaya}

Division of Hematology/ Oncology/ Stem Cell Transplantation, Columbia University Irving Medical Center, New York, USA

\section{David Gass}

Atrium Health/ Levine Children's Hospital, Charlotte, USA

\section{Rose McGee}

Cancer Predisposition Division, Oncology Department, St Jude Children's Research Hospital, Memphis, USA https://orcid.org/0000-0001-7392-6515

\section{Oz Mordechai}

Department of Pediatric Hematology Oncology, Rambam Health Care Campus, Haifa, Israel https://orcid.org/0000-0002-4340-9290

\section{Daniel Bowers}

UT Southwestern https://orcid.org/0000-0002-3947-2481

\section{Ted Laetsch}

Division of Oncology and Center for Childhood Cancer Research, Children's Hospital of Philadelphia, and Perelman School of Medicine at the University of Pennsylvania, Philadelphia, Pennsylvania, U

\section{Alexander Lossos}

Department of Oncology, Leslie and Michael Gaffin Center for Neuro-Oncology, Hadassah-Hebrew University Medical Center, Jerusalem, Israel

\section{Deborah Blumenthal}


Neuro-Oncology Service, Tel-Aviv Medical Center, Sackler Faculty of Medicine, Tel Aviv University, TelAviv, Israel

\section{Tomasz Sarosiek}

Lux Med Onkologia, Warsaw, Poland

\section{Lee Yen}

Department of Neurosurgery, Neurological Institute, Taipei Veterans General Hospital, Taipei, Taiwan

\section{Jeffrey Knipstein}

PRA Health Sciences https://orcid.org/0000-0002-0632-2751

\section{Anne Bendel}

Children's of Minnesota https://orcid.org/0000-0002-2062-0974

\section{Lindsey Hoffman}

Phoenix Children's Hospital, Phoenix, Arizona, USA

\section{Sandra Luna-Fineman}

Department of Pediatrics, Anschutz Medical Campus, Children's Hospital of Colorado, Colorado , USA

\section{Stefanie Zimmermann}

University Hospital Frankfurt, Paediatric Haematology and Oncology, Frankfurt, Germany

\section{Isabelle Scheers}

Pediatric Gastroenterology, Hepatology and Nutrition Unit, Cliniques Universitaires St Luc, Brussels, Belgium

\section{Kim Nichols}

St. Jude Children's Research Hospital https://orcid.org/0000-0002-5581-6555

\section{Michal Zapotocky}

Department of Pediatric Hematology and Oncology, Second Faculty of Medicine, University Hospital Motol, Charles University, Prague, Czech Republic

\section{Jordan Hansford}

Royal Children's Hospital https://orcid.org/0000-0001-7733-383X

\section{John Maris}

University of Pennsylvania and Children's Hospital of Philadelphia https://orcid.org/0000-0002-80887929

\section{Peter Dirks}

Hospital for Sick Children https://orcid.org/0000-0001-5718-6465

\section{Michael Taylor}

Sickkids Hospital

\section{Abhaya Kulkarni}

University of Toronto https://orcid.org/0000-0002-1706-7004

\section{Manohar Shroff}

Department of Diagnostic Imaging, The Hospital for Sick Children, Toronto, Canada

\section{Derek Tsang}

Princess Margaret Cancer Centre, University Health Network https://orcid.org/0000-0002-9762-6901 


\section{David Malkin}

The Hospital for Sick Children https://orcid.org/0000-0001-5752-9763

\section{Anita Villani}

Division of Haematology Oncology, The Hospital for Sick Children, Toronto, Canada

\section{Melyssa Aronson}

Zane Cohen Centre for Digestive Diseases, Mount Sinai Hospital, Toronto, Canada

\section{Carol Durno}

Zane Cohen Centre for Digestive Diseases, Mount Sinai Hospital, Toronto, Canada

\section{Adam Shlien}

Hospital for Sick Children

\section{Gad Getz}

Broad Institute https://orcid.org/0000-0002-0936-0753

\section{Yosef Maruvka}

Borad Institute

\section{Pamela Ohashi}

Princess Margaret Cancer Centre

\section{Cynthia Hawkins}

Hospital for Sick Children https://orcid.org/0000-0003-2618-4402

\section{Trevor Pugh}

Ontario Institute for Cancer Research

\section{Eric Bouffet}

Hospital for Sick Children

\section{Article}

Keywords: Mismatch repair, DNA polymerase, hypermutation, microsatellite instability, immune checkpoint inhibition, cancer, CMMR, Lynch, glioma

Posted Date: February 15th, 2021

DOI: https://doi.org/10.21203/rs.3.rs-155292/v1

License: (c) (i) This work is licensed under a Creative Commons Attribution 4.0 International License. Read Full License

Version of Record: A version of this preprint was published at Nature Medicine on January 6th, 2022. See the published version at https://doi.org/10.1038/s41591-021-01581-6. 
1 Mutation and Microsatellite Burden Predict Response to PD-1 Inhibition in Children

${ }^{\S}$ Daniel Morgenstern ${ }^{1,6},{ }^{\S}$ Sumedha Sudhaman ${ }^{2,49},{ }^{\S}$ Anirban Das ${ }^{1,2,49,59}$, Ailish Coblentz ${ }^{3}$, Jiil Chung ${ }^{2,49,62}$, Simone Stone ${ }^{4}$, Noor Alsafwani ${ }^{5,60}$, Vanja Cabric ${ }^{1}$, Liana Nobre ${ }^{1,49}$, Vanessa Bianchi $^{2,49}$, Melissa Edwards ${ }^{2,49}$, Lauren Sambira ${ }^{1}$, Shlomi Constantini ${ }^{7}$, Rina Dvir ${ }^{8}$, Michal Yalon-Oren ${ }^{9}$, Gadi Abebe Campino ${ }^{9}$, Shani Caspi ${ }^{9}$, Valerie Larouche ${ }^{10}$, Alyssa Reddy ${ }^{11}$, Michael Osborn ${ }^{12}$, Gary Mason ${ }^{13}$, Scott Lindhorst ${ }^{14}$, Annika Bronsema ${ }^{15}$, Vanan Magimairajan ${ }^{16}$, Enrico Opocher ${ }^{17}$, Rebecca Loret De Mola ${ }^{18}$, Magnus Sabel ${ }^{19}$, Charlotta Frojd $^{19}$, David Sumerauer ${ }^{20}$, David Samuel ${ }^{21}$, Kristina Cole ${ }^{22}$, Stefano Chiaravalli ${ }^{23}$, Maura Massimino $^{23}$, Patrick Tomboc ${ }^{24}$, David Ziegler ${ }^{25}$, Ben George ${ }^{26}$, An Van Damme ${ }^{27}$, Nobuko Hijiya $^{28}$, David Gass ${ }^{29}$, Rose McGee ${ }^{30}$, Oz Mordechai ${ }^{31}$, Daniel C. Bowers ${ }^{32}$, Ted Laetsch ${ }^{33}$, Alexander Lossos ${ }^{34}$, Deborah T. Blumenthal ${ }^{35}$, Tomasz Sarosiek ${ }^{36}$, Lee Yi Yen ${ }^{37}$, Jeffrey Knipstein $^{38}$, Anne Bendel ${ }^{39}$, Lindsey Hoffman $^{40}$, Sandra Luna-Fineman ${ }^{41}$, Stefanie Zimmermann ${ }^{42}$, Isabelle Scheers ${ }^{43}$, Kim E. Nichols ${ }^{44}$, Michal Zapotocky ${ }^{20}$, Jordan R. Hansford ${ }^{45}$, John M. Maris ${ }^{33}$, Peter Dirks ${ }^{46,47,49}$, Michael D. Taylor ${ }^{46,47,49}$, Abhaya V. 17 Kulkarni $^{46,48}$, Manohar Shroff ${ }^{3}$, Derek S. Tsang ${ }^{50}$, Anita Villani ${ }^{1,6}$, Melyssa Aronson ${ }^{51}$, Carol 18 Durno $^{51}$, Adam Shlien ${ }^{2,61}$, David Malkin ${ }^{1,2,6}$, Gad Getz ${ }^{52,53}$, Yosef E. Maruvka ${ }^{54}$, Pamela S. 19 Ohashi $^{4,55}$, Cynthia Hawkins ${ }^{49,56,61}$, Trevor J. Pugh ${ }^{57,58}$, Eric Bouffet ${ }^{1,6}$, *Uri Tabori ${ }^{1,2,6,49}$.

$\S \mathrm{DM}, \mathrm{SS}$ and $\mathrm{AD}$ are joint first authors and contributed equally to the work.

Number of pages: 40

Figures: 6. Tables: 0

Extended Data: Figures: 9. Tables: 3

Word Count: Summary: 197; Text: 3500

Keywords: Mismatch repair, DNA polymerase, hypermutation, microsatellite instability, immune checkpoint inhibition, cancer, CMMR, Lynch, glioma

\section{Corresponding author:}

$31 *$ Uri Tabori, MD

Staff Haematologist/Oncologist, Division of Haematology/Oncology.

The Hospital for Sick Children. 555 University Avenue, Toronto, ON, Canada, M5G 1X8. Professor of Paediatrics and Medical Biophysics, University of Toronto.

Senior Scientist, The Arthur and Sonia Labatt Brain Tumour Research Centre.

Tel: (416) 813-7654, ext. 201503, Fax: (416) 813-5327

37 E-mail: uri.tabori@sickkids.ca 
1. Division of Haematology Oncology, The Hospital for Sick Children, Toronto, Canada

2. Program in Genetics and Genome Biology, The Hospital for Sick Children, Toronto, Canada

3. Department of Diagnostic Imaging, The Hospital for Sick Children, Toronto, Canada

4. Princess Margaret Cancer Centre, University Health Network, Toronto, Canada

5. Division of Neuropathology, Department of Laboratory Medicine and Pathobiology, University of Toronto, Canada

6. Department of Paediatrics, University of Toronto, Toronto, Canada

7. Department of Paediatric Neurosurgery, Dana Children's Hospital, Tel-Aviv, Israel

8. Department of Pediatric Haematology-Oncology, Tel-Aviv Sourasky Medical Centre, Tel-Aviv, Israel

9. Department of Paediatric Haematology-Oncology, Sheba Medical Centre, Ramat Gan, Israel

10. Haematology/Oncology Centre Hospitalier Universitaire de Quebec, Quebec, Canada

11. Departments of Neurology and Paediatrics, University of California, San Francisco, California, USA

12. Women's and Children's Hospital, North Adelaide, Australia

13. Department of Paediatrics, University of Pittsburgh School of Medicine, Pittsburgh, USA

14. Neuro-Oncology, Department of Neurosurgery, and Department of Medicine, Division of Haematology/Medical Oncology, Medical University of South Carolina, Charleston, USA

15. Department of Paediatric Haematology and Oncology, University Medical Centre Hamburg-Eppendorf, Hamburg, Germany

16. Department of Paediatric Haematology-Oncology, Cancer Care Manitoba; Research Institute in Oncology and Haematology, University of Manitoba, Winnipeg, Canada

17. Paediatric Haematology, Oncology and Stem Cell Transplant Division, Padua University Hospital, Padua, Italy

18. Oregon Health and Science University, Portland, Oregon, USA

19. Department of Paediatrics, Institute of Clinical Sciences, Sahlgrenska Academy, University of Gothenburg \& Queen Silvia Children's Hospital, Sahlgrenska Univeristy Hospital, Gothenburg, Sweden

20. Department of Paediatric Haematology and Oncology, Second Faculty of Medicine, University Hospital Motol, Charles University, Prague, Czech Republic

21. Department of Paediatric Oncology, Valley Children's Hospital, Madera, California, USA

22. Division of Oncology and Centre for Childhood Cancer Research, Children's Hospital of Philadelphia, Department of Paediatrics, University of Pennsylvania School of Medicine, Pennsylvania, USA

23. Paediatric Unit, Fondazione IRCCS Istituto Nazionale dei Tumori, Milano, Italy

24. Department of Paediatrics, Ruby Memorial Hospital, West Virginia University, West Virginia, USA

25. Kids Cancer Centre, Sydney Children's Hospital, Randwick, New South Wales, and School of Women's and Children's Health, UNSW Sydney, Sydney, NSW, Australia

26. Division of Haematology and Oncology, Department of Medicine, Medical College of Wisconsin, Milwaukee, Milwaukee, Wisconsin, USA

27. Department of Paediatric Haematology and Oncology, Saint Luc University Hospital, Université Catholique de Louvain, Brussels Belgium

28. Division of Haematology/Oncology/Stem Cell Transplantation, Columbia University Irving Medical Centre, New York, USA

29. Atrium Health/ Levine Children's Hospital, Charlotte, USA

30. Cancer Predisposition Division, Oncology Department, St Jude Children's Research Hospital, Memphis, USA

31. Department of Paediatric Haematology Oncology, Rambam Health Care Campus, Haifa, Israel

32. Department of Paediatrics, The University of Texas Southwestern Medical School, Dallas, USA

33. Division of Oncology and Centre for Childhood Cancer Research, Children's Hospital of Philadelphia, and Perelman School of Medicine at the University of Pennsylvania, Philadelphia, Pennsylvania, USA

34. Department of Oncology, Leslie and Michael Gaffin Centre for Neuro-Oncology, Hadassah-Hebrew University Medical Centre, Jerusalem, Israel

35. Neuro-Oncology Service, Tel-Aviv Medical Centre, Sackler Faculty of Medicine, Tel Aviv University, TelAviv, Israel

36. Lux Med Onkologia, Warsaw, Poland

37. Department of Neurosurgery, Neurological Institute, Taipei Veterans General Hospital, Taipei, Taiwan

38. Division of Paediatric Haematology/ Oncology/ BMT, Medical College of Wisconsin, Milwaukee, Wisconsin, USA.

39. Department of Paediatric Haematology-Oncology, Children's Hospitals and Clinics of Minnesota Minnesota, USA

40. Phoenix Children's Hospital, Phoenix, Arizona, USA

41. Department of Paediatrics, Anschutz Medical Campus, Children's Hospital of Colorado, Colorado, USA 
97 42. University Hospital Frankfurt, Paediatric Haematology and Oncology, Frankfurt, Germany

98 43. Paediatric Gastroenterology, Hepatology and Nutrition Unit, Cliniques Universitaires St Luc, Brussels,

99 Belgium

100 44. Division of Cancer Predisposition, St Jude Children's Research Hospital, Memphis, USA

101 45. Children's Cancer Centre, Royal Children's Hospital, Murdoch Children's Research Institute; Department of

102 Paediatrics, University of Melbourne, Parkville, Victoria, Australia

103 46. Division of Neurosurgery, The Hospital for Sick Children, Toronto, ON, Canada

104 47. Developmental and Stem Cell Biology Program, The Hospital for Sick Children, Toronto, Canada

105 48. Child Health Evaluative Sciences, Research Institute, The Hospital for Sick Children, Toronto, Canada

106 49. The Arthur and Sonia Labatt Brain Tumour Research Centre, The Hospital for Sick Children, Toronto, Canada

107 50. Radiation Medicine Program, Princess Margaret Cancer Centre, Toronto, Canada

108 51.Zane Cohen Centre for Digestive Diseases, Mount Sinai Hospital, Toronto, Canada

109 52. Massachusetts General Hospital Centre for Cancer Research, Charlestown, Massachusetts, USA.

110 53. Broad Institute of Harvard and MIT, Cambridge, Massachusetts, USA

111 54. Biotechnology and Food Engineering. Tehcnion. Haifa. Israel.

112 55. Department of Immunology, University of Toronto, Canada

113 56. Department of Paediatric Laboratory Medicine, The Hospital for Sick Children, Toronto, Canada

114 57. Ontario Institute for Cancer Research, Princess Margaret Cancer Centre, Toronto, Canada

115 58. Department of Medical Biophysics, University of Toronto, Toronto, Canada

116 59. Department of Paediatric Haematology/ Oncology, Tata Medical Centre, Kolkata, India

117 60. Department of Pathology, College of Medicine, Imam Abdulrahman Bin Faisal University (IAU), Dammam, 118 Saudi Arabia

119 61. Department of Laboratory Medicine and Pathobiology, Faculty of Medicine, University of Toronto, Toronto,

$120 \quad$ Canada

121 62. Institute of Medical Science, Faculty of Medicine, University of Toronto, Toronto, Canada 


\section{Abstract}

123 Cancers arising from germline DNA mismatch-repair or polymerase-proofreading deficiencies

124 (MMRD and PPD) in children harbour the highest mutational and microsatellite 125 insertion/deletion (MS-indel) burden in humans and are lethal due to inherent resistance to 126 chemo-irradiation. Although immune checkpoint inhibitors (ICI) have failed to benefit children 127 in previous studies, we hypothesized that hypermutation caused by MMRD and PPD will 128 improve outcomes following ICI in these patients. ICI treatment of 45 progressive/recurrent 129 tumours from 38 patients revealed durable objective responses in the majority, culminating in 130 3-year survival of $41.4 \%$. High mutation burden predicted response for ultra-hypermutant 131 cancers ( $>100$ mutations/Mb) enriched for combined MMRD+PPD, while MS-indels predicted 132 response in MMRD tumours with lower mutation burden (10-100 mutations/Mb). Further, both 133 mechanisms were associated with increased immune infiltration even in "immunologically134 cold" tumours such as gliomas, contributing to the favorable response. Pseudo-progression 135 (flare) was common and associated with immune activation in both the tumour 136 microenvironment and systemically. Further, patients with flare continuing ICI treatment 137 achieved durable responses. Our study demonstrates improved survival for patients with 138 tumours not previously known to respond to ICI, including CNS and synchronous cancers, and 139 identifies the dual roles of mutation burden and MS-indels in predicting sustained responses to 140 immunotherapy. 
Accurate DNA replication in eukaryotic cells is ensured by the DNA polymerases Pol

$143 \delta$ and Pol $\varepsilon$, which control base incorporation and proofreading, and the mismatch repair

144 (MMR) system that undertakes post-replication surveillance. ${ }^{1}$ Germline and somatic mutations 145 in POLD1 and POLE (termed polymerase-proofreading deficiency: PPD), or the MMR genes 146 (MLH1, MSH2, MSH6, PMS2) result in DNA replication repair deficiency (RRD). RRD is a 147 major driver of hypermutation and microsatellite instability (MSI) in several adult and pediatric 148 cancers. ${ }^{2,3}$ Both germline PPD,${ }^{4}$ and monoallelic germline pathogenic variants in the MMR genes (Lynch syndrome) $)^{5}$ lead to adult-onset gastrointestinal and genitourinary cancers. ${ }^{6}$ In contrast, biallelic loss of MMR function in the germline causes constitutional mismatch repair deficiency (CMMRD) syndrome, a highly penetrant and aggressive cancer-predisposing condition. Affected individuals typically develop cancers at a young age, most commonly malignant gliomas, gastrointestinal and haematological malignancies. ${ }^{7,8}$ These cancers are frequently chemo-resistant, resulting in poor survival for affected patients. Indeed, individuals with CMMRD rarely survive beyond early adulthood. ${ }^{7}$ The burden of CMMRD cancers is significant in areas with high consanguinity, ${ }^{9}$ including many developing countries and among indigenous populations.

RRD cancers are universally hypermutant due to the continuous acquisition of multiple somatic mutations. Tumour mutation burden (TMB) of cancers driven by germline RRD is 100 to 1000 times higher than MMR-intact pediatric cancers. ${ }^{10}$ Further, many MMRD tumours acquire a secondary somatic mutation in POLD1/POLE leading to combined MMR+PPD, characterised by ultra-hypermutation $\left(>100\right.$ mutations/Mb). ${ }^{2}$ As a result, RRD cancers harbour the highest $\mathrm{TMB}$ observed among all human cancers. ${ }^{10}$ Hypermutant cancers such as melanoma and lung cancer, which are driven by ultraviolet light and smoking, respectively, respond to immune checkpoint inhibitors (ICI) ${ }^{10-12}$ targeting programmed death 1 (PD-1)

166 signalling. ${ }^{13}$ However, despite the dramatic anti-tumour effects reported in several 167 hypermutant adult cancers, ${ }^{14-16}$ these responses are sustained in only a subset of patients. ${ }^{17,18}$ 168 The role of TMB in determining the nature and duration of response is not well-established ${ }^{19-}$ 16921 . Other studies have also raised questions regarding the role of TMB and PD-ligand 1 (PD170 L1) expression as robust biomarkers of response to ICI. ${ }^{20,22-24}$ In contrast, MMR-deficient 171 colorectal carcinomas are responsive to ICI due to excess MSI ${ }^{25-28}$, suggesting that biomarkers

172 like $\mathrm{TMB}^{29}$, MSI or PD-L1 expression may not be individually sufficient to drive immune 173 responses following ICI across diverse cancer types ${ }^{19,21}$. 
Most cancers, including hypermutant brain tumours are considered 'immunologically cold' and are unresponsive to ICI. ${ }^{30}$ Importantly, ICI did not result in significant responses in multiple large pediatric clinical trials and is considered ineffective in the management of solid tumours in childhood and adolescence. ${ }^{24,31-34}$ Finally, for all solid tumours receiving immunotherapy, the distinction between true tumour progression and an inflammatory pseudoprogression is a major challenge and a barrier to effective therapy ${ }^{35-38}$.

Despite the lack of response to ICI observed in children in previous studies, we hypothesized that cancers originating from germline RRD may benefit from ICI due to their excess mutational load ${ }^{12}$. We further postulated that cancers driven by MMRD-only, PPD, or combined RRD (MMRD+PPD) will respectively exert their own unique mutational spectrum, driving local and systemic immune reactions, which would help shed light on the mechanisms of both response and pseudo-progression following ICI.

To address these hypotheses, we conducted a large, registry-based study, leveraging systematically-collected data gathered both retrospectively and prospectively through the International RRD Consortium ${ }^{2,7,10,2,39}$. This enabled us to evaluate outcomes and predictors of response to anti-PD-1 therapy in children with cancers driven by germline RRD. Uniquely, this also provided us the opportunity to investigate the efficacy of ICI in individuals with synchronous malignancies who are otherwise excluded from conventional clinical trials.

\section{Results} between May 2015 and March 2019 and followed by the International RRD Consortium registry study group (Methods and Extended Data Table S1). While guidelines for treatment were provided by the consortium, and regular communication was organized with the treating team for ongoing radiological monitoring, response assessment, and management of adverse effects (Methods), the patients were not treated prospectively as part of a clinical trial. The PD1 inhibitor used was either nivolumab $(n=34,75 \%)$ or pembrolizumab $(n=11,25 \%)$, as per availability and choice of the treating team. All patients had germline RRD, diagnosed as CMMRD ( $\mathrm{n}=28,74 \%)$, Lynch $(\mathrm{n}=8,21 \%)$, or PPD $(\mathrm{n}=2,5 \%)$ syndromes (Extended Data Table S1). Median age at treatment was 12.1 years (range: $3.1-28.1$ ) for patients with CMMRD, and 15.7 years (range: $8.5-43.4)$ for those with Lynch syndrome $(p=0.07)$. Seven cancer types were included and classified into 3 major groups: central nervous system (CNS) tumours $(n=31$, 69\%; disseminated: 2, 6\%), non-CNS solid tumours ( $\mathrm{n}=11,24 \%$; disseminated: 7, 64\%), and haematological malignancies $(n=3,7 \%)$ (Fig.1a). The majority $(n=43,93 \%)$ of cancers were 
progressive/recurrent after failure of first-line therapy (Extended Data Table S1). Three patients with gastrointestinal cancers received ICI directly following surgery: two of whom had synchronous CNS tumours and one who had metastatic disease. Data cutoff for outcomes was October 2019.

Responses and/or stable disease were observed in 25/45 (55.5\%) tumours, with most of the responses $(n=20 ; 80 \%)$ being sustained at a median follow-up of 1.87 years at the time of this analysis. Central radiological review (RANO and RECIST criteria; Methods) ${ }^{40,41}$ revealed complete response in $6(17 \%)$, partial response in $9(25 \%)$, stable disease in $7(19 \%)$, and progressive disease in $14(39 \%)$ (Fig.1b,c). Among the 7 patients with synchronous malignancies, responses in both tumours were seen in one patient, and at least in one tumour in 4 patients (Fig.2a). The three patients with haematological malignancies had progressed at a median time of 4.5 months after starting ICI therapy.

Of note, $12(27 \%)$ tumours exhibited early radiological findings of edema and enhancement, suggestive of peri-tumoural inflammation or tumour progression (Fig.1b,d and 2a). This phenomenon occurred at a median of 34 days (range 7-74) from treatment initiation and has been termed tumour 'flare.' ${ }^{42}$ These patients presented with acute clinical deterioration with headache, bone or abdominal pain, depending on the location of their tumours. Eight patients (6 with CNS and 2 with non-CNS solid tumours) stopped therapy and died. Importantly, 4 patients ( 3 with CNS and 1 with non-CNS solid tumour) who continued to receive ICI with adequate supportive care subsequently demonstrated objective responses. Because this suggested pseudo-progression, ${ }^{35,38,43}$ these tumours were studied in more detail.

Estimated 3-year overall survival (OS) was 41.4\% (95\% CI: 38.5, 44.2) (Fig.2b), with $18(47 \%)$ patients being alive at the time of last follow-up (Fig.2a). This is noteworthy considering the refractory nature of their cancers. Analysis by cancer type revealed that nonCNS solid tumours had a significantly better survival compared to CNS tumours (Fig.2c and Extended Data Fig.1d; $p=0.01)$. Nevertheless, the OS of 39.3\%, (95\% CI; 36.3, 42.3) and PFS of $26.9 \%(95 \% \mathrm{CI} ; 23.2,30.6)$ for patients with recurrent/progressive CNS tumours is a dramatic improvement compared to the historically rapidly fatal outcomes (Fig.2c,d and Extended Data Fig.1c) $)^{7,9,12,44}$. All patients with non-CNS solid tumours continuing ICI had durable responses and are alive at a median follow-up of 2.6 years (range: 0.38-3.5). Remarkably, 13 patients with CNS tumours continued to survive for an additional 9.6 months (median; range: 1.5-27) after radiological progression (Fig.2a,d and Extended Data Table S1). Collectively, these data suggest that late and continued responses to immunotherapy possibly due to the dynamic clonal evolution and obligatory mutation accumulation in RRD cancers ${ }^{10}$. 


\section{Molecular determinants of response to immunotherapy}

To better understand the molecular determinants of response of RRD cancers to ICI, biopsy specimens and blood samples were collected before and during therapy from the patients for centralized analysis (Methods). Whole exome analysis of tumours ( $n=39$, Fig.3) revealed high variability in the number of single nucleotide variants (SNV), including ultrahypermutation (median: 233.8 mutations/Mb, range: 3.4-912), which was associated with tumour genotype. MMRD-only cancers $(n=16)$ had significantly fewer SNVs (median: 15.8 mutations $/ \mathrm{Mb}$ ) than MMRD+PPD cancers $(\mathrm{n}=23$, median 391.4 mutations $/ \mathrm{Mb} ; \mathrm{p}<0.0001$; Fig.4b). This correlated with germline status, as 21 (67.7\%) cancers in CMMRD patients harboured MMRD+PPD (median: 398.98 mutations/Mb), while all cancers in individuals with Lynch syndrome lacked somatic PPD (median: 21.76 mutations $/ \mathrm{mb} ; \mathrm{p}=0.03$ ). Both cancers originating from germline PPD, one colorectal carcinoma (P13; ICI.29) and one glioblastoma (P17; ICI.33) had acquired somatic MMRD resulting in ultra-hypermutation (Fig.2a and 3).

Analysis of COSMIC signatures, which reflect the imprints of the underlying RRD mutational processes, ${ }^{45,46}$ revealed that signatures 6 and 26 were enriched in MMRD-only cancers, and that all tumours in patients with Lynch syndrome exhibited signature 6. In contrast, signatures 14 and 15 were frequent in cancers in patients with CMMRD, and signature 14 was enriched in tumours with MMRD+PPD, highlighting the unique and potential diagnostic role of these signatures in determining germline predisposition in RRD cancers. ${ }^{45}$

As questions exist regarding the contribution of tumour-intrinsic characteristics such as mutation load and MS-indels to ICI response, we sought to determine whether response is modulated by a dynamic interaction between these factors. ${ }^{47} \mathrm{TMB}$ correlated significantly with both response and survival. This was true for tumours with higher total SNVs/Mb ( $\mathrm{p}=0.003$, Fig.4a), as well as non-synonymous variants, synonymous variants and total indels (Extended Data Fig.2a-d). Both response and survival were also associated with a higher tumour neoantigen load (Extended Data Fig.2e). Remarkably, response and survival were predicted by RRD status. Cancers with MMRD+PPD (median: 392.7 mutations/Mb) had higher likelihood of response compared with cancers with MMRD alone (median: 15.7 mutations/Mb) $(\mathrm{p}<0.0001)$ (Fig.4b).

Recent work has shown that response in MMRD tumours is related to the degree of genome-wide MS-indels. ${ }^{3,48,49}$ Across our entire cohort, total MS-indels, calculated by MSmutect (Methods), ${ }^{50}$ were not predictive of tumour response, but still predicted improved patient survival (Fig.4c). We did not find a significant association between high MS-indel and 
TMB ( $\mathrm{p}=0.47$ ), thereby suggesting independent roles for both carcinogenic mechanisms for likely different tumour subsets. We therefore hypothesized that MS-indels are immunogenic in MMRD-only cancers, where the contribution of TMB is less dominant. To test this, we analyzed MMRD-only and MMRD+PPD tumours separately (Fig.4d). In MMRD-only cancers, total MS-indels were higher in responders than in non-responders, and were significantly associated with survival ( $\mathrm{p}=0.025$, Fig.4d). This was not observed in MMRD+PPD cancers with high TMB (Fig.4d). Further, in MMRD-only cancers, high TMB failed to predict response $(\mathrm{p}=0.52)$. Combining the prediction models for both these types of RRD cancers (MMRD-only and MMRD+PPD), high SNVs and total MS-indels together strongly predicted improved survival (Fig.4e, $\mathrm{p}=0.0024$ ).

To determine whether SNVs and MS-indels drive response within a more homogeneous cancer type, we interrogated both these genomic signatures in CNS tumours. Both components contributed independently to the response and survival (Extended Data Fig.3c,g). In multivariate analysis (Methods), high mutation burden remained the most significant predictor for response $(p=0.01)$ and OS $(p=0.05)$ (Extended Data Table S2 and S3), highlighting the important contribution of a combined MMRD+PPD status in determining response to ICI. Collectively, these data suggest dual roles for TMB and MS-indels in determining immunotherapy responses in RRD cancers.

\section{Immune microenvironment and response to therapy in childhood RRD cancers}

Next, we examined whether the RRD subgroups affect the tumour micro-environment and response to therapy. We tested multiple immune markers using immunohistochemistry for immune cell infiltration (CD3, CD4, CD8 and CD68) and checkpoint ligand (PD-L1) expression (Methods, Fig.5 and Extended Data Fig.4). All immune markers were scored blindly by our central pathologist ( $\mathrm{CH}$, Methods).

PD-L1 expression correlated with both response and improved survival $(p=0.04$, Fig.5a). Overall, increased lymphocytic infiltration within the tumour microenvironment correlated with response (Extended Data Fig.4a,b). Specifically, high CD8-T-cell infiltration predicted both response and improved OS ( $p=0.0002$, Fig.5b). All non-CNS solid tumours including those with MMRD-only, harboured high MSI, exhibited high CD8+T-cell infiltration (Extended Data Fig.5a,b), and responded to ICI. (Extended Data Fig.5a-c). This corroborates previous reports in which MMR-deficient gastrointestinal tumours had high CD8+ T-cell infiltration. ${ }^{51}$ High T-cell infiltration was also observed in CNS tumours which are traditionally considered an 'immune-privileged' site. ${ }^{52} \mathrm{CNS}$ tumours with a high mutation burden and 
MMRD+PPD not only had increased CD8+ T-cell infiltration, but also significantly higher

311 expression of PD-L1 ( $<<0.04$; Fig.5c). This suggests that in the setting of ultra-hypermutation

312 driven by combined MMRD+PPD and high genomic MS-indels, the increased activation of

313 the immune microenvironment can explain the remarkable responses seen in the CNS tumours.

314 Indeed, in these CNS tumours, high CD8+T-cell infiltration was associated with improved OS

315 (Extended Data Fig.6a,b). Collectively, tumours exhibiting high expression of immune 316 markers and a favorable genomic profile (high SNVs or MS-indels) had a 3-year OS of 87.8\%

317 (95\% CI; 84, 91.5) as compared to $33.2 \%(95 \% \mathrm{CI} ; 27.2,39.2)$ for tumours lacking these

318 biomarkers ( $\mathrm{p}=0.005$, Fig.5d).

\section{Tumour flare is an immune reaction to therapy}

To determine etiology of the flare phenomenon, we first analyzed the genomic and immune markers of these tumours. Cancers developing flare had pre-treatment genomic and immune characteristics similar to the responders without flare (Methods; Extended Data Fig.7a-d). We then compared the pre- and on-therapy tumours in two patients who had further surgical debulking at flare. Transcriptomic analysis and immune inference using single state deconvolution (Methods) revealed an increase in the overall immune cell expression at flare (Fig.6a,b). Notably, transcriptome signaling revealed that activated CD8+ T-cells were significantly increased in both samples following ICI. Using T-cell receptor clonotype analysis (TCR, Fig.6c,d), we observed a dramatic increase of T-cell repertoire in the post-treatment samples as compared to their baseline. One sample demonstrated an increase in both clonality and diversity of the T-cell population, with some original clones expanding during flare (Fig.6c), whereas for the other sample, there was reduction in diversity but significantly heightened clonality in the T-cell population (Fig6d). Additionally, both increased CD8+ Tcell infiltration and PD-L1 expression were observed in both tumours during flare when compared to their pretreatment samples (Fig.6e,f and Extended Data Fig.8a,b). These observations suggest a pre-existing (specific) immune response and further ICI-driven (nonspecific and specific) intra-tumoral immune expansion at flare.

We next investigated whether this immune activation could be observed systemically. We performed serial flow cytometry analysis of blood samples from multiple patients, prior to treatment initiation and within the first 90 days of initiation of ICI (Methods). Flare was associated with an expansion of peripheral CD8+T-cells expressing Ki67 (a marker of proliferation) and 4-1BB (a member of the TNF receptor family) (Extended Data Fig.9a-d and

343 Fig.6g). Detection of Ki67+ CD8+ T-cells has been previously reported to be associated with 
superior response to PD-1 blockade. ${ }^{53} 4-1 \mathrm{BB}$ is well-known as a marker of T-cell activation and has co-stimulatory activity for activated T-cells. ${ }^{54,55}$ Flare was associated with higher proportion of 4-1BB $+\mathrm{CD} 8+\mathrm{T}$-cells in the peripheral blood as compared to non-responders, as well as responders without flare (Fig.6h). Studies have previously shown that 4-1BB+CD8+ T-cells correlate with response to PD-1 blockade. ${ }^{56}$ Importantly, we did not find an increase in the 4-1BB+CD4+ T-cell population (Extended Data Fig.9e,f), supporting our hypothesis that CD8+ T-cells expressing 4-1BB reflect the expansion of a tumour-specific response. Taken together with the immune profile of the tumour microenvironment, we conclude that flare following PD-1 blockade is associated with proliferation of tumour-antigen reactive T-cells.

\section{Discussion}

Our study reveals dramatic responses to PD-1 blockade and an associated improved survival for relapsed/refractory hypermutant cancers in children and young adults with germline DNA replication repair deficiency. Several insights can be derived from the sustained responses in different tumour types, and the contributions of TMB, MS-indels and the microenvironment to both response and flare.

The unique responses to ICI in children with germline RRD are different from previous observations by several groups. First, our data starkly contrast with the lack of ICI efficacy in childhood cancers in general ${ }^{32-34}$, as well as specifically for progressive paediatric brain tumours ${ }^{57}$. The lack of response in paediatric cancers, which is independent of PD-L1 expression $^{24}$ or systemic immune activation ${ }^{24,33}$, has been attributed to the low mutation burden $^{2,10,12}$, a different immunomodulatory role of the gut microbiome ${ }^{58}$ at a very young age $^{24,32,34}$, low expression of major histocompatibility complex ${ }^{59}$ and the predominance of macrophages in the tumour micro-environment ${ }^{60,61}$. Some of these causes may need to be reexamined in view of our data.

Second, more than half of RRD paediatric CNS tumours had partial to complete objective responses, resulting in a median survival of 2 years when ICI was continued. This is remarkable, as historically, rapid progression with a median post-relapse survival of merely 2.6 months has been reported in children with RRD high-grade glioma. ${ }^{62}$ In contrast, hypermutant gliomas in adults fail to respond to ICI. These are different from RRD gliomas as they harbour relatively lower TMB with late acquisition of sub-clonal mutations during tumorigenesis possibly due to treatment-related secondary MMR deficiency, which is associated with less MS-indels, and lack of robust neoantigens. ${ }^{63-65}$ This is accompanied by multi-layered immunosuppression with a non-inflamed tumour microenvironment dominated 
by a myeloid infiltrate, exhausted T-cell phenotype, and secretion of suppressive 379 cytokines/molecules and hostile physical factors (hypoxia, acidosis and nutrient 380 deprivation $)^{63,64}$.

381 Third, all patients who continued ICI therapy for non-CNS solid tumours including 382 disseminated cancers responded and are alive at a median follow-up of 2.6 years. This 383 compares favourably with MMR-deficient cancers in older patients (median age: 60 years, versus 12.3 years in our study), in whom late failures were noted, resulting in 50-55\% survival at 2 years. ${ }^{26,27,66,67}$

Collectively, the dramatic responses and favourable outcome observed in childhood RRD cancers can be explained by several key biological features. First, the earlier onset ${ }^{10}$, combined with significantly higher mutations ${ }^{2}$, MS-indels ${ }^{3}$, and neoantigen burden drive CD8+T-cell activation which is especially robust in children and adolescents when compared to older patients ${ }^{68-70}$. Second, the additional loss of the polymerase-proofreading mechanism confers genomic mutational signatures (both MS-signatures ${ }^{3}$ and COSMIC signatures 10, 20, and 14) which may play unique immunogenic role in determining response and survival. Third, the exceptionally high rate of obligatory and continuous accumulation of mutations in combined MRRD+PPD cancers ${ }^{10}$ likely confers ongoing immunogenicity, contributing to immune-surveillance leading to both the durable and the delayed responses observed in our cohort.

Additional insights from this study include the ongoing and delayed responses observed in tumours that progressed on ICI therapy. This resulted in the difference between progressionfree (post-ICI) and overall survival for patients who continued treatment after a second progression while on ICI therapy (Fig.2d and Extended Data Fig.1b,d). Additionally, responses in a tissue-agnostic manner were seen in a patient with synchronous tumours exhibiting favourable genomic and immune biomarkers for response (P01). As cancer immune surveillance is different than the irreversible resistance which occurs upon progression after chemo-radiation approaches, these data support the exploration of neoadjuvant ${ }^{71,72}$, maintenance, and combinatorial uses of ICI in these patients, to limit toxicities and improve effectiveness of first-line strategies.

There is increasing evidence that responses to ICI cannot be predicted by single biomarker. ${ }^{73,74,19,75}$ Our data confirm that this is true in RRD cancers which are driven by dysfunction in both SNV and MS-indel repair. Although initially TMB was thought to be the sole contributor to immune response in hypermutant cancers ${ }^{22}$, indels and MS-indels have been suggested to be the main drivers of response to ICI in MMR-deficient cancers ${ }^{3,49,76}$. We add a 
412 new dimension to this concept by revealing that in cancers driven by MMRD-only, TMB is 413 relatively lower, and MS-indels drive response, while in MMRD+PPD cancers, the role of MS-

414 indels is attenuated, and TMB is the major driver of response. The dual roles of both mutational 415 mechanisms also affect the microenvironment, with upregulation of PD-L1 and infiltration of 416 CD8+ T-cells. Importantly, the combination of both genomic mechanisms and immune 417 markers are powerful predictors of survival in RRD cancers and should be incorporated as 418 combined biomarkers in future clinical trials.

419 Paradoxically, this hyperactivation of the immune microenvironment can be 420 detrimental, because tumour flare, which indicates both specific (pre-existing) and non-specific 421 (new) expansion of TCR clones, can be misinterpreted as tumour progression and lead to 422 premature treatment abandonment. Flare is quite common in RRD cancers, especially when 423 tumour burden is high. ${ }^{12}$ These flare responses are very different from hypermutant adult 424 glioblastomas originating as a result previous chemo-radiotherapy ${ }^{65}$ where hyper-progression 425 is reported. ${ }^{37,77}$ Our data support the development of novel functional imaging techniques ${ }^{78}$ and 426 minimally invasive biomarkers ${ }^{79,80}$ to better predict tumour response, and innovative strategies 427 to modulate the immune response. This is important, as continuation of immunotherapy can 428 result in late responses and long-term survival for patients developing tumour flare.

429 Despite the limitations of a registry study, this is the first description of a large cohort 430 of children and young adults with previously recurrent/progressive fatal germline DNA 431 replication repair deficient cancers demonstrating impressive responses to PD-1 blockade. ${ }^{81}$ 432 Although longer follow-up is required to determine whether immunotherapy can be a curative 433 strategy for RRD cancers, the sustained responses and lack of late relapses in a significant 434 number of patients in this cohort are encouraging. This study also sheds light on the complex 435 interplay between the tumour genomic status, microenvironment, and the systemic immune 436 response, especially in the context of extreme mutation and MS-indel burdens. Future trials 437 should prospectively analyse the roles of germline versus somatic RRD, and the components 438 of the RRD machinery, to identify patients who are likely to derive maximal benefit from anti439 PD-1 immunotherapy. Lastly, our study highlights the impact of studying a genetic cancer 440 syndrome to understand general cancer processes ${ }^{82}$ and deriving direct therapeutic implications 441 for patients. 
442 Acknowledgements: This research is supported by a Stand Up to Cancer- Bristol-Meyers 443 Squibb Catalyst Research Grant (Grant Number: SU2C-AACR-CT07-17), which is 444 administered by the American Association for Cancer Research, the scientific partner of 445 SU2C. This work is also supported by an Enabling Studies Program grant from BioCanRx 446 Canada's Immunotherapy Network (a Network Centre of Excellence), the Canadian Institutes 447 for Health Research (CIHR) grant (PJT-156006), and the CIHR Joint Canada-Israel Health 448 Research Program. Additional financial support was provided by Meagan's Walk Foundation, 449 LivWise Foundation, the Zane Cohen Center, BRAINchild Foundation, St. Baldrick's 450 Foundation International Scholar Award (with generous support from the Team Campbell 451 Foundation), and Guglietti We Love You Connie Foundation.

\section{Author contributions:}

454 DM, UT and EB planned the study. AD and UT wrote the manuscript. AC and MS reported 455 the radiology. NA and $\mathrm{CH}$ reported the pathology. SS, AS and TP were responsible for the 456 bioinformatics analysis. JC, YM and GG were responsible for the microsatellite indel analysis. 457 SS and PO were responsible for the flow cytometry studies. VB and ME were involved in 458 patient enrolment and sample coordination. Those involved in patient management included 459 EB, DM, VC, LFN, LS, SC, MO, RD, GAC, SC, VL, AR, MO, GM, SL, AB, VM, EO, RLDM, 460 AD, MS, CF, DS, DS, KC, SC, MM, PT, DZ, BG, AVD, NH, DG, RM, KEN, MZ, OM, DM, 461 TL, AL, DM, TS, LYY, JK AB, LH, SLF, SZ, IS, DS, PD, MT, AK, DT, DM, AV, MA, CD, 462 MA and UT. All authors have reviewed and agreed to the contents of the manuscript.

464 Competing Interests: None 
ONLINE METHODS

\section{Study design and patients}

Patients included in this retrospective registry study were identified through the International Replication Repair Deficiency Consortium (IRRDC), based at the Hospital for Sick Children (SickKids), Toronto, that has enrolled 200 patients from 45 countries since 2007. Patients with confirmed or suspected RRD were eligible to participate. Institutional ethics board approval was obtained. Consent was obtained from patients and families for participation in the study. This included submission of clinical and imaging data, as well as tissue and blood samples for centralized analysis. Germline diagnosis of CMMRD, Lynch or PPD were confirmed by the genetic counsellor from the consortium. This was done based on the results of the family history, next-generation panel sequencing of germline samples for $M M R$ and $P O L$ genes (performed locally or centrally at CLIA-approved laboratories), and immunohistochemical (IHC) staining pattern of the tumour and normal tissues (performed at the Hospital for Sick children, Toronto). Thirty-eight patients with 45 cancers who had received treatment with anti-PD1-directed immune checkpoint inhibitor (ICI) therapy were identified and are reported here. Guidelines for ICI treatment were provided by the consortium. Regular communication was organized with the treating team to discuss clinical responses, along with ongoing radiological monitoring, as well as management of adverse effects of ICI. Monitoring for toxicity and stopping rules were as per the recommendations provided by an ongoing clinical trial (NCT02992964). However, patients were not treated prospectively as part of a clinical trial. Ultimately, the choice of the agent and the nuances of therapy remained at the discretion of the treating team. Blood samples were collected prospectively throughout the course of ICI therapy at specified time-points.

Clinical records were reviewed to obtain details regarding patient demographics, cancer diagnosis, date of initiation and completion of ICI, choice of ICI agent, and survival outcomes (including date of disease progression and/or patient death). Imaging at baseline and following initiation of ICI were obtained for central review of objective tumour response. For the remaining cases, response (or otherwise) was determined by the assessment of the local treating team. Available scans were centrally reviewed by a radiologist blind to the clinically determined response, and tumour measurements were documented according to the RANO criteria. ${ }^{40}$ Briefly, the best tumour response was determined as the percentage change in the product of bi-perpendicular dimensions from baseline on the contrast-enhanced T1 images.

497 Complete response (CR) was defined as the disappearance of all enhancing disease (measurable and non-measurable) sustained for at least 4 weeks, with stable or improved non- 
enhancing FLAIR/T2W lesions, no new lesions and clinical stability. Partial response (PR) was defined as $50 \%$ or more decrease of all measurable enhancing lesions sustained for at least 4 weeks, with no progression of non-measurable disease, stable or improved non-enhancing FLAIR/T2W lesions, no new lesions and clinical stability. Stable disease (SD) was defined as images that did not qualify for complete response, partial response, or progression, with stable non-enhancing FLAIR/T2W lesions and clinical stability. Progressive disease (PD) was defined as $25 \%$ or more increase in enhancing lesions, with increase (significant) in nonenhancing FLAIR/T2W lesions, not attributable to other non-tumour causes any new lesions, and clinical deterioration not attributable to other causes. For patients with non-CNS solid tumours, the revised RECIST (version 1.1) was used. ${ }^{41}$ CR was defined as complete disappearance, PR as at least $30 \%$ decrease in sum of the diameters, PD as at least $20 \%$ increase in the sum of the diameters of the target lesion, and SD as lack of sufficient change to be classified as $\mathrm{CR} / \mathrm{PR} / \mathrm{PD}$. Patients with objective radiological response (CR/PR) and/ or stable disease (SD) were labelled as 'responders.' Among those with progressive disease, patients experiencing rapid early clinical and/or radiological deterioration (with $>100 \%$ increase in tumour size within 90 days of starting ICI therapy) were defined as demonstrating a 'flare' response and were studied in more detail. For patients able to continue ICI, subsequent imaging was reviewed to confirm response or progression. ${ }^{43}$ Those with sustained clinical and/or radiological progression despite continuation of ICI treatment were classified as 'nonresponders.' For biomarker prediction analyses, 'responders' also included those with an initial 'flare,' who continued on ICI and demonstrated delayed responses.

\section{Whole exome sequencing (WES) and analysis}

Genomic DNA from 39 tumours, along with matched germline blood samples, was extracted using the PaxGene Blood DNA Extraction Kit (Cat No./ID: 761133) for blood samples, Qiagen DNeasy Blood \& Tissue Kits (Cat No./ID: 69504) for frozen tissue, MasterPure Complete DNA and RNA Purification Kit (Epicentre \#MC85200) for paraffin embedded tissue). WES was performed at The Centre for Applied Genomics (TCAG), SickKids, using SureSelect Agilent All Exon v5 kit, followed by sequencing (150X) on Illumina HiSeq 2500. The software bcl2fastq2 v2.17 was used to generate raw fastq files. Alignment to the hg38 reference genome, followed by pre-processing and QC was adapted from the GATK standard pipeline, using BWA-MEM 0.7.12 (alignment), BAMQC, Picard 2.6.0 (QC). Somatic variant calling was done post-alignment, using processed bam files from tumour and matched normal samples, to call both single nucleotide variants (SNVs) and 
insertion deletion (indel) variants. A consensus vcf file of shared variants across 2 or more of

5344 variant callers (Mutect v1.1.5, GATK v3.6/Mutect2, Strelka v1.0.14, and Varscan2 Somatic v2.4.2) was generated for SNVs and indels separately, using VCFtools 0.1.15, and these vcfs were annotated using VEP v83. The tumour mutation burden (SNVs per megabase) from WES data was calculated by counting total number of somatic SNVs divided by total number of callable bases in megabases $(\sim 50 \mathrm{Mb})$. DeconstructSigs ${ }^{83}$ was used to determine COSMIC signatures $^{46}$ in the mutation spectrum within a tri-nucleotide context for each sample. All bioinformatics analyses were performed on the SickKids High Performance Cluster (HPF) and the UHN High Performance Cluster for Health $(\mathrm{H} 4 \mathrm{H})$.

\section{Neoantigen calling}

The Mutect 2 vcfs generated for each tumour (described above) were used as input along with bioinformatically generated HLA-typing (using a consensus of HLAminer, and HLAVBSeq) of germline WES fastqs using MuPeXI,${ }^{84}$ to get a list of strong binding candidate neoantigens per HLA-type.

\section{Microsatellite (MS)-indel calling}

Microsatellite indels were called on the bam files of tumour and matched normal samples, using an in-house pipeline using MSMuTect. The detailed methods for this algorithm have been previously reported. ${ }^{50}$ Briefly, repeats of five or more nucleotides were considered to be MS loci, and using the PHOBOS algorithm and the lobSTR approach, tumour and normal BAM files were aligned with their 5' and 3' flanking sequences. Each MS-locus allele was estimated using the empirical noise model, which is the probability of observing a read with a microsatellite (MS) length $\mathrm{k}$ and motif $\mathrm{m}$, where the true length of the allele is $\mathrm{j}$ with the motif $\mathrm{m}$. This was used to call the MS alleles with the highest likelihood of being the true allele at each MS-locus. The MS alleles of each tumour and matched normal pair were called individually, which were compared to identify the mutations on the tumour MS-loci. The Akaike Information Criterion (AIC) score was assigned to both the tumour and normal models, and a threshold score that was determined by using simulated data was applied to make the final MS-indel call.

\section{Immunohistochemistry}

Four-micron thick sections of formalin-fixed paraffin-embedded (FFPE) surgical specimens were stained using an automated stainer (Dako OMNIS) with the following primary 
antibodies: PD-L1 (clone28-8, Abcam, 1/500), CD68 (Clone c8/144B, Dako OMNIS), CD8

568 (Clone c8/144B, Dako OMINS), CD3 (polyclonal rabbit, Dako OMNIS), and CD4 (Clone SP35, Sigma- Aldrich, 1/50). Quantitative evaluation of the immunohistochemical stains was performed by examining each section using at least five to seven different high-power fields with the most abundant tumour-infiltrating lymphocyte areas. The tumour was considered PDL1 positive if $\geq 1 \%$ of tumour cells exhibited a circumferential and/or partial linear plasma membrane PD-L1 staining of tumour cells at any intensity. ${ }^{85}$ The percentage of infiltrating immune system cells was estimated by manual eyeballing as none, mild, moderate, and severe $(0=$ none, $\quad<10 \%=$ mild, $10-50 \%=$ moderate, $>50 \%=$ severe $)$. For downstream analyses, infiltration higher than the median values of the continuous data of immune infiltrates was used to classify tumours as 'high' or 'low' infiltration for each marker.

\section{Immune inference analysis}

RNA was extracted as per standard kit protocol from tumours biopsied at both, baseline and time of flare, in 2 patients (P31 and P33) and submitted for total r-RNA depletion RNAsequencing on HiSeq 2500, at TCAG. Following sequencing, the raw data were analyzed using STAR aligner followed by RSEM expression analysis to generate a gene expression matrix for each sample. This was then run through CIBERSORT, ${ }^{86}$ a single state deconvolution algorithm, to generate immune inference data for 22 immune cell subtypes. The immune inference results from CIBERSORT were plotted using "ComplexHeatmap" ${ }^{87}$ package on R 3.5 .

\section{T-cell receptor rearrangement repertoire profiling}

Genomic DNA was extracted (methods as above) from tumours biopsied at both baseline and at time of flare in 2 patients (P31 and P33). These were transferred to the Pugh laboratory at the University Health Network (UHN) in Toronto, where library preparation and capTCRseq ${ }^{88}$ hybrid capture were performed. Following library preparation, the samples were sequenced first on a MiSeq for QC purposes and then 300ng of each sample, pooled in a ratio of 1:1:1, was processed for a 3 -step capture using target hybrid capture panel. ${ }^{88}$ Post-capture QC was performed on a MiSeq, followed by sequencing of up to a depth of $\sim 2$ millions reads on the NextSeq. Post-sequencing the raw data were analyzed using MiXCR version 2.1.12, 'iNext', 'immunarch' R packages and Pugh Lab customized functions on R version 3.5 to look at T-cell receptor rearrangements in the form of unique clonotypes (VDJ rearranged sequences) for T-cell receptors alpha, beta, gamma and delta. As the total read depth varied across the 
601 cohort, affecting the total successfully aligned reads, all raw fastq reads were down-sampled 602 to $\sim 1$ million reads. QC parameters of percent aligned reads, reads used in clonotypes, final

603 clonotype count and the total number of clonotypes per 1000 reads were considered.

604

\section{Flow cytometry}

Viable frozen peripheral blood mononuclear cells were incubated with Fc block (BD 607 Biosciences) prior to staining for surface markers (anti-CD3 - clone UCHT1, anti-CD4 - clone 608 RPA-T4, anti-CD8 - clone RPA-T8, anti-4-1BB- clone 4B4-1, anti-TIGIT 609 clone MBSA43, anti-Ki67 - clone 20Raj1) and viability dye (eBioscience). Cells were fixed 610 and permeabilized for intercellular staining with the Foxp3 transcription factor staining buffer 611 set (BD). Flow cytometry voltages were set using Rainbow beads (Spherotech) with the same 612 setting between experiments. Samples were acquired on a BD LSR Fortessa flow cytometer 613 and data were analyzed using the FlowJo software.

614

615 Statistical analyses and reproducibility

616 Overall survival (OS) and event-free survival (EFS) was estimated using Kaplan-Meier 617 statistics and determined from the date of initiation of ICI therapy. Patients without an event 618 were censored at the date of last known contact. Uniquely in this population, several patients 619 had multiple separate synchronous malignancies and therefore in these analyses, survival is 620 presented for each individual cancer/tumour in addition to analyses per patient. For example, 621 for the tumour-wise analysis, a patient experiencing an event related to one cancer diagnosis, would be shown as censored (rather than an event) for a second synchronous cancer diagnosis. Multivariate analysis was performed using binary logistic regression analysis for independent variables for predicting response. Cox regression analysis was performed for predictors of overall survival. Variables used in multivariate analysis included age, gender, site, ICI agent, 626 higher mutations ( $\mathrm{SNV}$ s/Mb) and higher MS-indels (than the median). Statistical analyses were 627 performed with SPSS version 20, R version 3.5 and Python version 2.7. Statistical significance 628 was calculated using Welch's unequal variances t-test and the Wilcoxon-Mann-Whitney test, 629 for parametric and nonparametric data, respectively. All p-values were 2-sided, with a cut-off

630 of 0.05 for significance. Plots generated were edited for aesthetics using Adobe Illustrator 631 version 23.0.1. 
633 1. Cortez, D. Replication-Coupled DNA Repair. Molecular cell 74, 866-876 (2019).

634 2. Campbell, B.B., et al. Comprehensive Analysis of Hypermutation in Human Cancer.

3. Chung, J., et al. DNA polymerase and mismatch repair exert distinct microsatellite instability signatures in normal and malignant human cells. Cancer discovery (2020).

4. Mur, P., et al. Role of POLE and POLD1 in familial cancer. Genetics in medicine : official journal of the American College of Medical Genetics (2020).

5. Seppälä, T.T., et al. European guidelines from the EHTG and ESCP for Lynch syndrome: an updated third edition of the Mallorca guidelines based on gene and gender. The British journal of surgery (2020).

6. Lynch, H.T., Snyder, C.L., Shaw, T.G., Heinen, C.D. \& Hitchins, M.P. Milestones of Lynch syndrome: 1895-2015. Nature reviews. Cancer 15, 181-194 (2015).

7. Tabori, U., et al. Clinical Management and Tumour Surveillance Recommendations of Inherited Mismatch Repair Deficiency in Childhood. Clinical cancer research : an official journal of the American Association for Cancer Research 23, e32-e37 (2017).

8. Ripperger, T. \& Schlegelberger, B. Acute lymphoblastic leukemia and lymphoma in the context of constitutional mismatch repair deficiency syndrome. European journal of medical genetics 59, 133-142 (2016).

9. Amayiri, N., et al. High frequency of mismatch repair deficiency among pediatric high grade gliomas in Jordan. International journal of cancer 138, 380-385 (2016).

10. Shlien, A., et al. Combined hereditary and somatic mutations of replication error repair genes result in rapid onset of ultra-hypermutated cancers. Nat Genet 47, 257-262 (2015).

11. Finocchiaro, G., Langella, T., Corbetta, C. \& Pellegatta, S. Hypermutations in gliomas: a potential immunotherapy target. Discovery medicine 23, 113-120 (2017).

12. Bouffet, E., et al. Immune Checkpoint Inhibition for Hypermutant Glioblastoma Multiforme Resulting From Germline Biallelic Mismatch Repair Deficiency. Journal of clinical oncology : official journal of the American Society of Clinical Oncology 34, 2206-2211 (2016).

13. Wei, S.C., Duffy, C.R. \& Allison, J.P. Fundamental Mechanisms of Immune Checkpoint Blockade Therapy. Cancer discovery 8, 1069-1086 (2018).

14. Liu, D., et al. Integrative molecular and clinical modeling of clinical outcomes to PD1 blockade in patients with metastatic melanoma. Nature medicine 25, 1916-1927 (2019).

15. Kammerer-Jacquet, S.F., et al. Targeting the PD-1/PD-L1 Pathway in Renal Cell Carcinoma. International journal of molecular sciences 20(2019).

16. Garon, E.B., et al. Pembrolizumab for the treatment of non-small-cell lung cancer. $N$ Engl J Med 372, 2018-2028 (2015).

17. Topalian, S.L., et al. Five-Year Survival and Correlates Among Patients With Advanced Melanoma, Renal Cell Carcinoma, or Non-Small Cell Lung Cancer Treated With Nivolumab. JAMA oncology (2019).

18. Hellmann, M.D., et al. Nivolumab plus Ipilimumab in Advanced Non-Small-Cell Lung Cancer. The New England journal of medicine 381, 2020-2031 (2019).

19. Gurjao, C., Tsukrov, D., Imakaev, M., Luquette, L.J. \& Mirny, L.A. Limited evidence of tumour mutational burden as a biomarker of response to immunotherapy. bioRxiv, 2020.2009.2003.260265 (2020).

20. Marabelle, A., et al. Association of tumour mutational burden with outcomes in patients with advanced solid tumours treated with pembrolizumab: prospective biomarker analysis of the multicohort, open-label, phase 2 KEYNOTE-158 study. The Lancet. Oncology 21, 1353-1365 (2020). 
21. Bersanelli, M. Tumour mutational burden as a driver for treatment choice in resistant tumours (and beyond). The Lancet. Oncology 21, 1255-1257 (2020).

22. Yarchoan, M., Hopkins, A. \& Jaffee, E.M. Tumour Mutational Burden and Response Rate to PD-1 Inhibition. The New England journal of medicine 377, 2500-2501 (2017).

23. Cristescu, R., et al. Pan-tumour genomic biomarkers for PD-1 checkpoint blockadebased immunotherapy. Science (New York, N.Y.) 362(2018).

24. Geoerger, B., et al. Pembrolizumab in paediatric patients with advanced melanoma or a PD-L1-positive, advanced, relapsed, or refractory solid tumour or lymphoma (KEYNOTE-051): interim analysis of an open-label, single-arm, phase 1-2 trial. The Lancet. Oncology 21, 121-133 (2020).

25. Le, D.T., et al. PD-1 Blockade in Tumours with Mismatch-Repair Deficiency. The New England journal of medicine 372, 2509-2520 (2015).

26. Le, D.T., et al. Phase II Open-Label Study of Pembrolizumab in Treatment-Refractory, Microsatellite Instability-High/Mismatch Repair-Deficient Metastatic Colorectal Cancer: KEYNOTE-164. J Clin Oncol 38, 11-19 (2020).

27. André, T., et al. Pembrolizumab in Microsatellite-Instability-High Advanced Colorectal Cancer. The New England journal of medicine 383, 2207-2218 (2020).

28. Llosa, N.J., et al. The vigorous immune microenvironment of microsatellite instable colon cancer is balanced by multiple counter-inhibitory checkpoints. Cancer discovery 5, 43-51 (2015).

29. Schrock, A.B., et al. Tumour mutational burden is predictive of response to immune checkpoint inhibitors in MSI-high metastatic colorectal cancer. Ann Oncol 30, 10961103 (2019).

30. Sampson, J.H., Gunn, M.D., Fecci, P.E. \& Ashley, D.M. Brain immunology and immunotherapy in brain tumours. Nature reviews. Cancer 20, 12-25 (2020).

31. Pearson, A.D.J., et al. ACCELERATE and European Medicines Agency Paediatric Strategy Forum for medicinal product development of checkpoint inhibitors for use in combination therapy in paediatric patients. European journal of cancer (Oxford, England : 1990) 127, 52-66 (2020).

32. Geoerger, B., et al. Atezolizumab for children and young adults with previously treated solid tumours, non-Hodgkin lymphoma, and Hodgkin lymphoma (iMATRIX): a multicentre phase 1-2 study. The Lancet. Oncology 21, 134-144 (2020).

33. Merchant, M.S., et al. Phase I Clinical Trial of Ipilimumab in Pediatric Patients with Advanced Solid Tumours. Clinical cancer research : an official journal of the American Association for Cancer Research 22, 1364-1370 (2016).

34. Davis, K.L., et al. Nivolumab in children and young adults with relapsed or refractory solid tumours or lymphoma (ADVL1412): a multicentre, open-label, single-arm, phase 1-2 trial. The Lancet. Oncology 21, 541-550 (2020).

35. Martin-Romano, P., et al. Evidence of pseudoprogression in patients treated with PD1/PDL1 antibodies across tumour types. Cancer medicine 9, 2643-2652 (2020).

36. Okada, H., et al. Immunotherapy response assessment in neuro-oncology: a report of the RANO working group. The Lancet. Oncology 16, e534-e542 (2015).

37. Sasaki, A., et al. Predictive factors for hyperprogressive disease during nivolumab as anti-PD1 treatment in patients with advanced gastric cancer. Gastric cancer : official journal of the International Gastric Cancer Association and the Japanese Gastric Cancer Association 22, 793-802 (2019).

38. Ellingson, B.M., Chung, C., Pope, W.B., Boxerman, J.L. \& Kaufmann, T.J. Pseudoprogression, radionecrosis, inflammation or true tumour progression? challenges associated with glioblastoma response assessment in an evolving therapeutic landscape. Journal of neuro-oncology 134, 495-504 (2017). 
39. Bakry, D., et al. Genetic and clinical determinants of constitutional mismatch repair deficiency syndrome: report from the constitutional mismatch repair deficiency consortium. European journal of cancer (Oxford, England : 1990) 50, 987-996 (2014).

40. Wen, P.Y., et al. Updated response assessment criteria for high-grade gliomas: response assessment in neuro-oncology working group. Journal of clinical oncology : official journal of the American Society of Clinical Oncology 28, 1963-1972 (2010).

41. Eisenhauer, E.A., et al. New response evaluation criteria in solid tumours: revised RECIST guideline (version 1.1). European journal of cancer (Oxford, England : 1990) 45, 228-247 (2009).

42. Yoshida, T., Furuta, H. \& Hida, T. Risk of tumour flare after nivolumab treatment in patients with irradiated field recurrence. Medical oncology (Northwood, London, England) 34, 34 (2017).

43. Wen, P.Y., et al. Response Assessment in Neuro-Oncology Clinical Trials. Journal of clinical oncology : official journal of the American Society of Clinical Oncology 35, 2439-2449 (2017).

44. Guerrini-Rousseau, L., et al. Constitutional mismatch repair deficiency-associated brain tumours: report from the European C4CMMRD consortium. Neuro-oncology advances 1, vdz033 (2019).

45. Alexandrov, L.B., et al. The repertoire of mutational signatures in human cancer. Nature 578, 94-101 (2020).

46. Alexandrov, L.B., et al. Signatures of mutational processes in human cancer. Nature 500, 415-421 (2013).

47. Chen, J.A., Ma, W., Yuan, J. \& Li, T. Translational Biomarkers and Rationale Strategies to Overcome Resistance to Immune Checkpoint Inhibitors in Solid Tumours. Cancer treatment and research 180, 251-279 (2020).

48. Le, D.T., et al. Mismatch repair deficiency predicts response of solid tumours to PD-1 blockade. Science (New York, N.Y.) 357, 409-413 (2017).

49. Mandal, R., et al. Genetic diversity of tumours with mismatch repair deficiency influences anti-PD-1 immunotherapy response. Science (New York, N.Y.) 364, 485-491 (2019).

50. Maruvka, Y.E., et al. Analysis of somatic microsatellite indels identifies driver events in human tumours. Nature biotechnology 35, 951-959 (2017).

51. Gang, W., et al. Strategy to targeting the immune resistance and novel therapy in colorectal cancer. Cancer medicine 7, 1578-1603 (2018).

52. Carson, M.J., Doose, J.M., Melchior, B., Schmid, C.D. \& Ploix, C.C. CNS immune privilege: hiding in plain sight. Immunol Rev 213, 48-65 (2006).

53. Huang, A.C., et al. T-cell invigoration to tumour burden ratio associated with anti-PD1 response. Nature 545, 60-65 (2017).

54. Watts, T.H. TNF/TNFR family members in costimulation of T cell responses. Annual review of immunology 23, 23-68 (2005).

55. Chester, C., Sanmamed, M.F., Wang, J. \& Melero, I. Immunotherapy targeting 4-1BB: mechanistic rationale, clinical results, and future strategies. Blood 131, 49-57 (2018).

56. Clouthier, D.L., et al. An interim report on the investigator-initiated phase 2 study of pembrolizumab immunological response evaluation (INSPIRE). J Immunother Cancer 7, 72 (2019).

57. Blumenthal, D.T., et al. Pembrolizumab: first experience with recurrent primary central nervous system (CNS) tumours. Journal of neuro-oncology 129, 453-460 (2016).

58. Routy, B., et al. Gut microbiome influences efficacy of PD-1-based immunotherapy against epithelial tumours. Science (New York, N.Y.) 359, 91-97 (2018). 
59. Haworth, K.B., et al. Going back to class I: MHC and immunotherapies for childhood cancer. Pediatric blood \& cancer 62, 571-576 (2015).

60. Vakkila, J., Jaffe, R., Michelow, M. \& Lotze, M.T. Pediatric cancers are infiltrated predominantly by macrophages and contain a paucity of dendritic cells: a major nosologic difference with adult tumours. Clinical cancer research : an official journal of the American Association for Cancer Research 12, 2049-2054 (2006).

61. Majzner, R.G., et al. Assessment of programmed death-ligand 1 expression and tumour-associated immune cells in pediatric cancer tissues. Cancer 123, 3807-3815 (2017).

62. Amayiri, N., et al. High frequency of mismatch repair deficiency among pediatric high grade gliomas in Jordan. Int J Cancer (2015).

63. Khasraw, M., Reardon, D.A., Weller, M. \& Sampson, J.H. PD-1 inhibitors: Do they have a future in the treatment of glioblastoma? Clinical cancer research : an official journal of the American Association for Cancer Research (2020).

64. Khasraw, M., Walsh, K.M., Heimberger, A.B. \& Ashley, D.M. What is the Burden of Proof for Tumour Mutational Burden in gliomas? Neuro-Oncology (2020).

65. Touat, M., et al. Mechanisms and therapeutic implications of hypermutation in gliomas. Nature 580, 517-523 (2020).

66. Azad, N.S., et al. Nivolumab Is Effective in Mismatch Repair-Deficient Noncolorectal Cancers: Results From Arm Z1D-A Subprotocol of the NCI-MATCH (EAY131) Study. Journal of clinical oncology : official journal of the American Society of Clinical Oncology 38, 214-222 (2020).

67. Marabelle, A., et al. Efficacy of Pembrolizumab in Patients With Noncolorectal High Microsatellite Instability/Mismatch Repair-Deficient Cancer: Results From the Phase II KEYNOTE-158 Study. Journal of clinical oncology : official journal of the American Society of Clinical Oncology 38, 1-10 (2020).

68. Zhao, J., et al. Immune and genomic correlates of response to anti-PD-1 immunotherapy in glioblastoma. Nature medicine 25, 462-469 (2019).

69. Thomas, R., Wang, W. \& Su, D.M. Contributions of Age-Related Thymic Involution to Immunosenescence and Inflammaging. Immun Ageing 17, 2 (2020).

70. Simon, A.K., Hollander, G.A. \& McMichael, A. Evolution of the immune system in humans from infancy to old age. Proc Biol Sci 282, 20143085 (2015).

71. Cloughesy, T.F., et al. Neoadjuvant anti-PD-1 immunotherapy promotes a survival benefit with intratumoural and systemic immune responses in recurrent glioblastoma. Nature medicine 25, 477-486 (2019).

72. Schalper, K.A., et al. Neoadjuvant nivolumab modifies the tumour immune microenvironment in resectable glioblastoma. Nature medicine 25, 470-476 (2019).

73. Blank, C.U., Haanen, J.B., Ribas, A. \& Schumacher, T.N. CANCER IMMUNOLOGY. The "cancer immunogram". Science (New York, N.Y.) 352, 658-660 (2016).

74. Bruni, D., Angell, H.K. \& Galon, J. The immune contexture and Immunoscore in cancer prognosis and therapeutic efficacy. Nature reviews. Cancer 20, 662-680 (2020).

75. Bai, R., Lv, Z., Xu, D. \& Cui, J. Predictive biomarkers for cancer immunotherapy with immune checkpoint inhibitors. Biomark Res 8, 34 (2020).

76. Turajlic, S., et al. Insertion-and-deletion-derived tumour-specific neoantigens and the immunogenic phenotype: a pan-cancer analysis. The Lancet. Oncology 18, 1009-1021 (2017).

77. Ellingson, B.M., et al. Estimated clinical efficacy and radiographic response characteristics of PD1 inhibition in newly diagnosed and recurrent glioblastoma in clinical practice: A report from the iRANO Working Group. Journal of Clinical Oncology 38, 2521-2521 (2020). 
78. Antonios, J.P., et al. Detection of immune responses after immunotherapy in glioblastoma using PET and MRI. Proceedings of the National Academy of Sciences of the United States of America 114, 10220-10225 (2017).

79. Kim, K.H., et al. The First-week Proliferative Response of Peripheral Blood PD$1(+)$ CD8(+) T Cells Predicts the Response to Anti-PD-1 Therapy in Solid Tumours. Clinical cancer research : an official journal of the American Association for Cancer Research 25, 2144-2154 (2019).

80. Georgiadis, A., et al. Noninvasive Detection of Microsatellite Instability and High Tumour Mutation Burden in Cancer Patients Treated with PD-1 Blockade. Clinical cancer research : an official journal of the American Association for Cancer Research 25, 7024-7034 (2019).

81. Pasello, G., et al. Real world data in the era of Immune Checkpoint Inhibitors (ICIs): Increasing evidence and future applications in lung cancer. Cancer treatment reviews 87, 102031 (2020).

82. Tomasetti, C., Li, L. \& Vogelstein, B. Stem cell divisions, somatic mutations, cancer etiology, and cancer prevention. Science (New York, N.Y.) 355, 1330-1334 (2017).

83. Rosenthal, R., McGranahan, N., Herrero, J., Taylor, B.S. \& Swanton, C. DeconstructSigs: delineating mutational processes in single tumours distinguishes DNA repair deficiencies and patterns of carcinoma evolution. Genome biology 17, 31 (2016).

84. Bjerregaard, A.M., Nielsen, M., Hadrup, S.R., Szallasi, Z. \& Eklund, A.C. MuPeXI: prediction of neo-epitopes from tumour sequencing data. Cancer immunology, immunotherapy: CII 66, 1123-1130 (2017).

85. Ionescu, D.N., Downes, M.R., Christofides, A. \& Tsao, M.S. Harmonization of PD-L1 testing in oncology: a Canadian pathology perspective. Current oncology (Toronto, Ont.) 25, e209-e216 (2018).

86. Newman, A.M., et al. Robust enumeration of cell subsets from tissue expression profiles. Nature methods 12, 453-457 (2015).

87. Gu, Z., Eils, R. \& Schlesner, M. Complex heatmaps reveal patterns and correlations in multidimensional genomic data. Bioinformatics (Oxford, England) 32, 2847-2849 (2016).

88. Mulder, D.T., et al. CapTCR-seq: hybrid capture for T-cell receptor repertoire profiling. Blood advances 2, 3506-3514 (2018). 
a

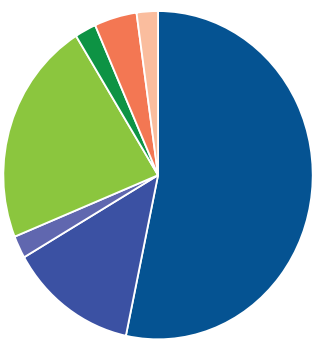

Glioma Grade IV (WHO) $(\mathrm{n}=24)$

$\square$ Glioma Grade III (WHO) $(n=6)$

$\square$ CNS Embryonal tumor $(n=1)$

$\square$ Gastrointestinal Cancers $(n=10)$

$\square$ Urothelial Cancer $(n=1)$

$\square$ T-cell Leukemia/Lymphoma $(\mathrm{n}=2)$

$\square$ Acute Myeloid Leukemia $(n=1)$ b

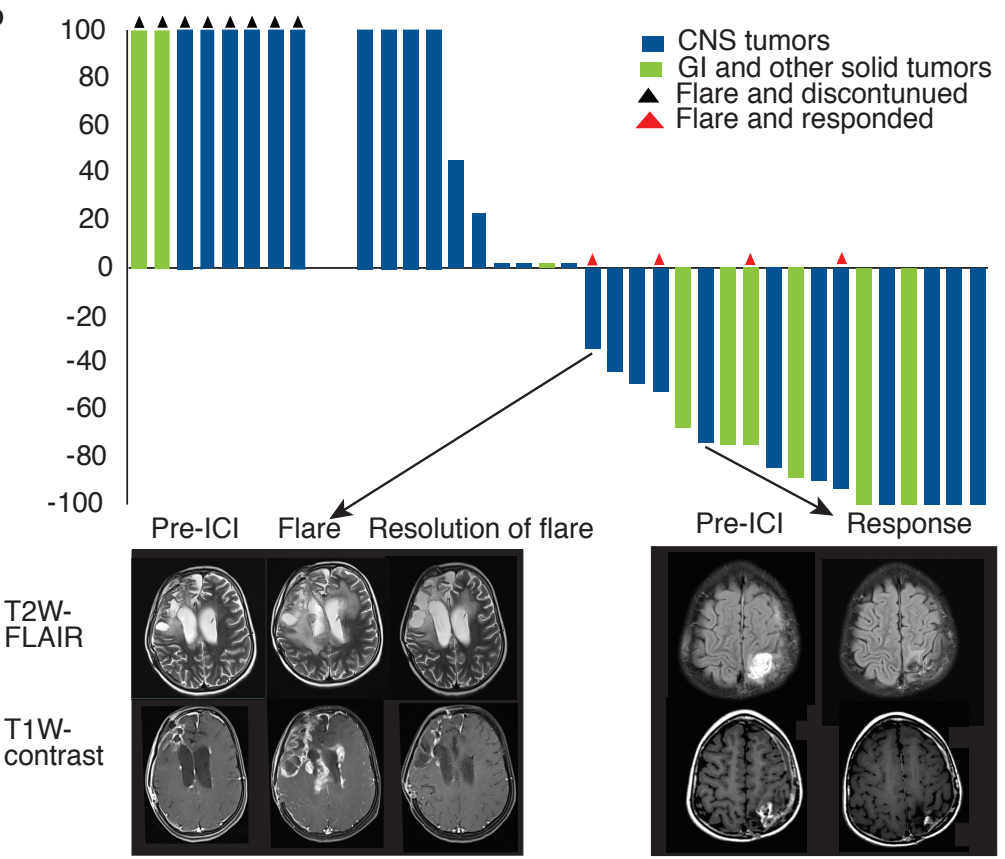

867 Fig. 1. Clinical response to ICI across cancer types in patients with germline DNA

868 replication repair deficiency. (a) Distribution of tumour types across 38 patients who

869 developed 45 tumours. (b) Waterfall plot of all radiological responses in non-haematological

870 malignancies. Values show the best fractional change in the 2 dimensions from baseline

871 measurements as per RANO and RECIST criteria. Arrows point to representative T2-weighted

872 FLAIR and T1-weighted contrast-enhanced MRI sequences in two patients showing flare and 873 partial responses. 

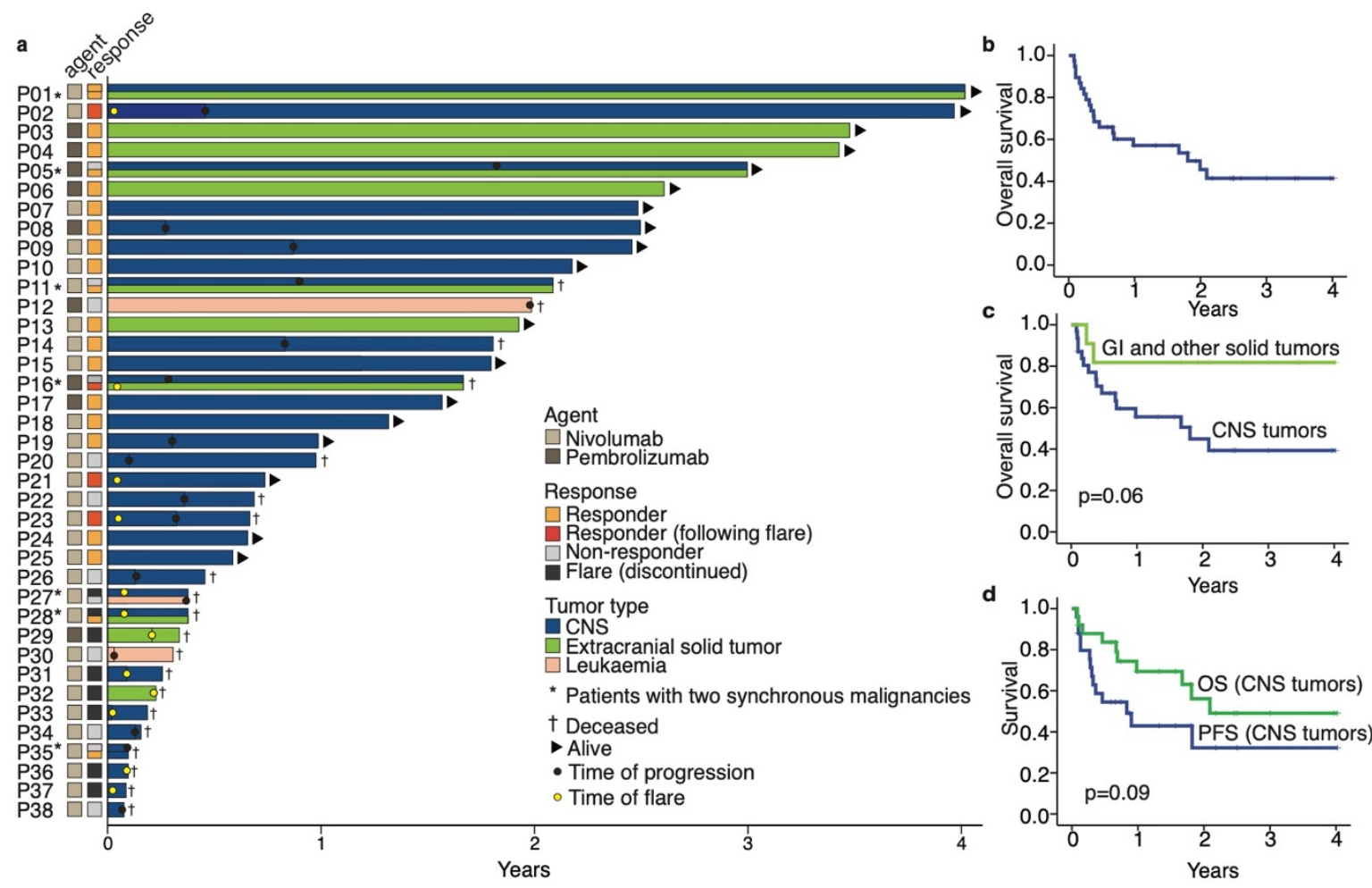

875 Fig.2. Patient outcome and survival by tumour type. (a) Swimmer plot by patient and

876 tumour type. (b) Kaplan-Meier (KM) estimates of overall survival for all patients (c) KM

877 estimates of overall survival as per tumour type. Median survival for CNS tumours was 21.6

878 months. Median survival was not reached for non-CNS solid tumours. (d) KM estimates of

879 progression free and overall survival for CNS tumours continuing ICI therapy. Note: prolonged

880 median survival at 24 months (estimated 3-year OS=49.1\%) despite initial radiological 881 progression at a median of 9.9 months (estimated 3-year PFS=32\%). 


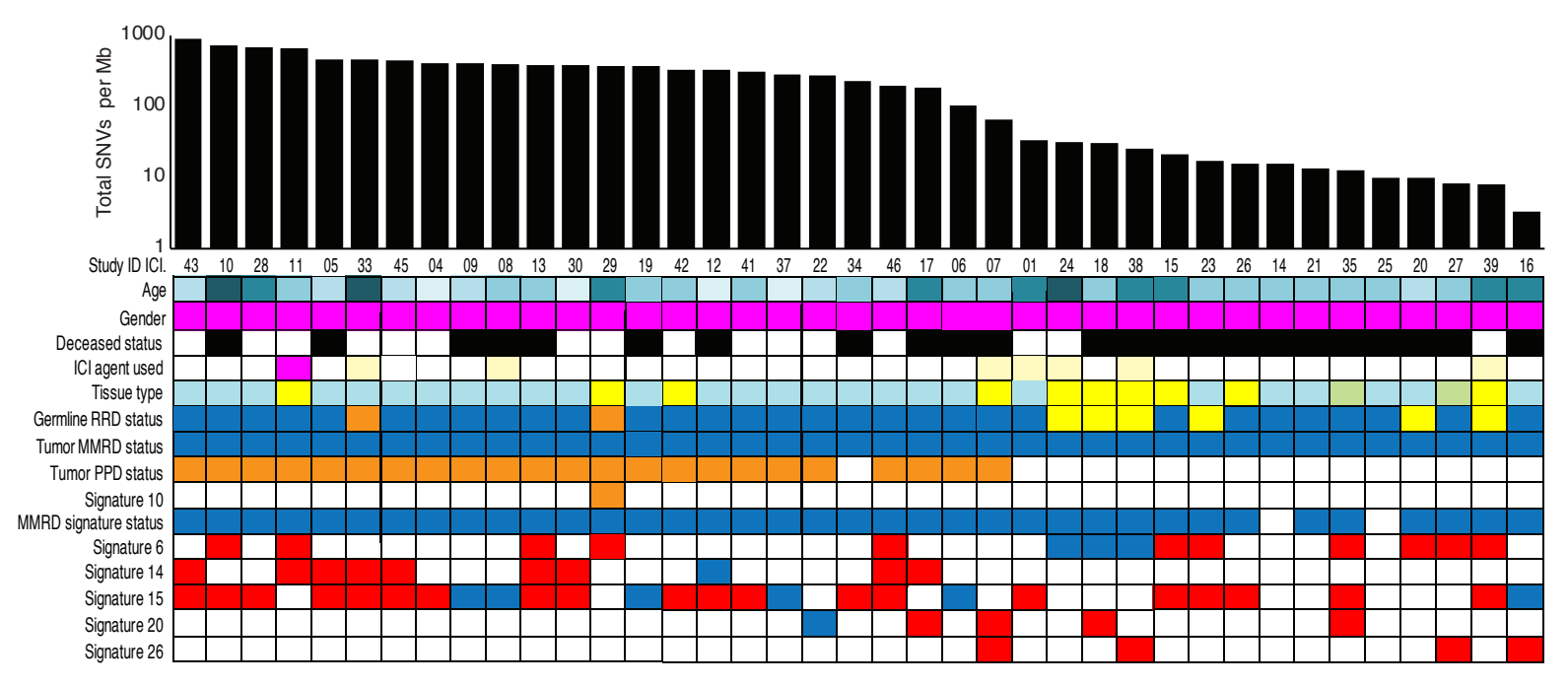

882

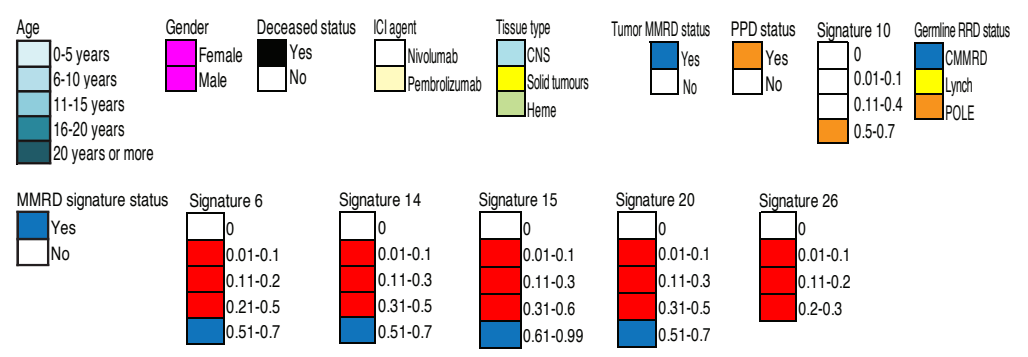

883 Fig. 3. Genomic biomarkers, survival and response to PD1 blockade. (a) Onco-plot

884 summarising the genomic features from 39 available paired tumour and germline exomes, and

885 their clinical correlates. (b) Response and overall survival (OS) by single nucleotide variants

886 (SNVs) per Mb. For survival analysis, median SNV burden was used. (c) SNVs as a function

887 of MMRD (blue) and MMRD+PPD (orange) status (left), and response association with both

888 SNV and RRD status. (c) Response and overall survival (OS) by microsatellite indels (MS-

889 indels). For survival analysis, median MS-indel values were used. (d) Response and overall

890 survival by total MS-indel count for MMRD and MMRD+PPD cancers separately. (e) Kaplan-

891 Meier (KM) estimates using combined SNVs/Mb and MS-indel in all RRD cancers.

892 (Abbreviations: MMRD: mismatch-repair deficiency; PPD: Polymerase-proofreading

893 deficiency; MS-indel: microsatellite insertion/ deletion.) 

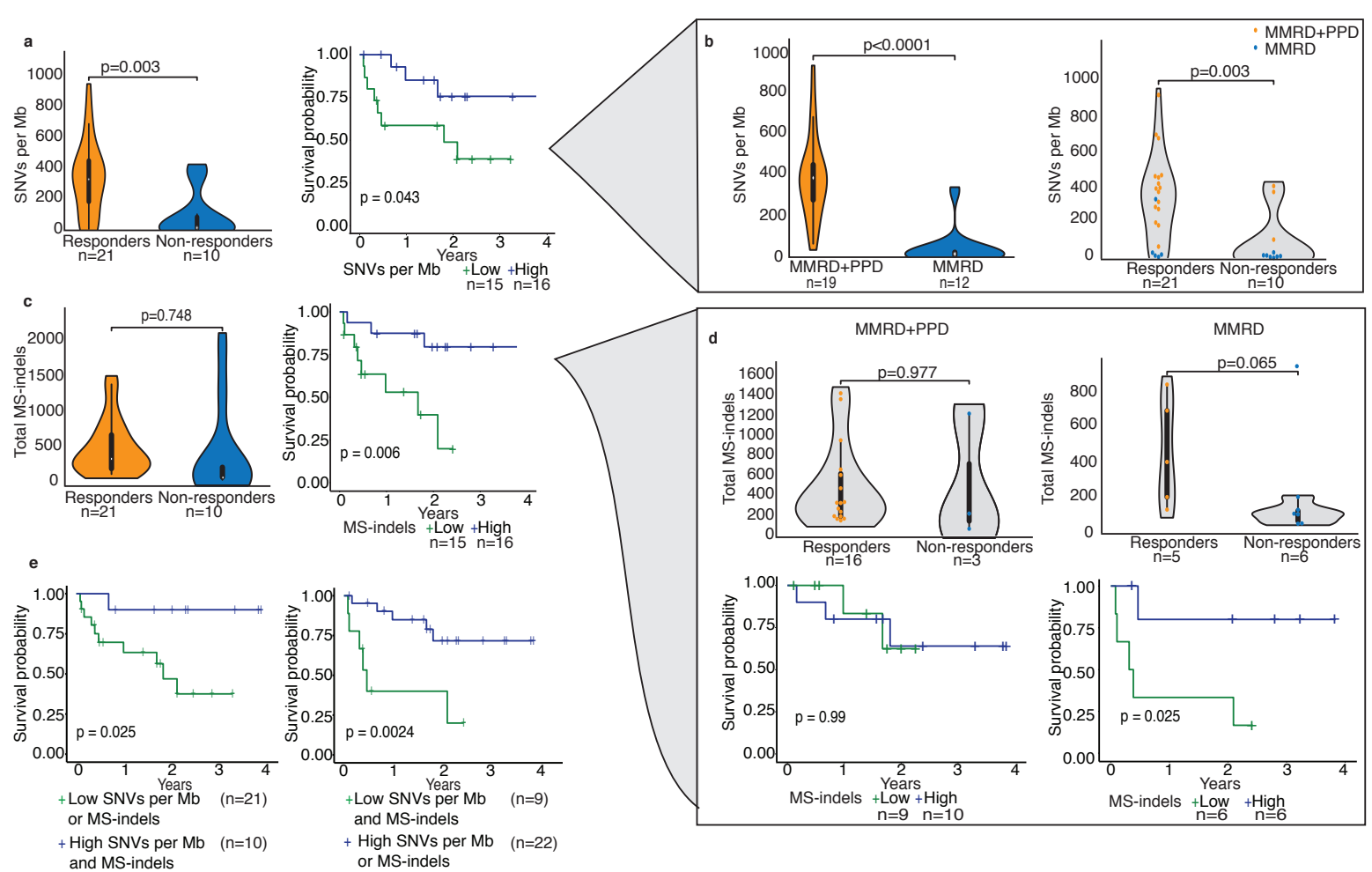

894

895 Fig. 4. Genomic biomarkers, survival and response to PD1 blockade. (a) Onco-plot

896 summarising the genomic features from 39 available paired tumour and germline exomes, and

897 their clinical correlates. (b) Response and overall survival (OS) by single nucleotide variants

898 (SNVs) per Mb. For survival analysis, median SNV burden was used. (c) SNVs as a function 899 of MMRD (blue) and MMRD+PPD (orange) status (left), and response association with both 900 SNV and RRD status. (c) Response and overall survival (OS) by microsatellite indels (MS901 indels). For survival analysis, median MS-indel values were used. (d) Response and overall 902 survival by total MS-indel count for MMRD and MMRD+PPD cancers separately. (e) Kaplan903 Meier (KM) estimates using combined SNVs/Mb and MS-indel in all RRD cancers. 904 (Abbreviations: MMRD: mismatch-repair deficiency; PPD: Polymerase-proofreading 905 deficiency; MS-indel: microsatellite insertion/ deletion.) 

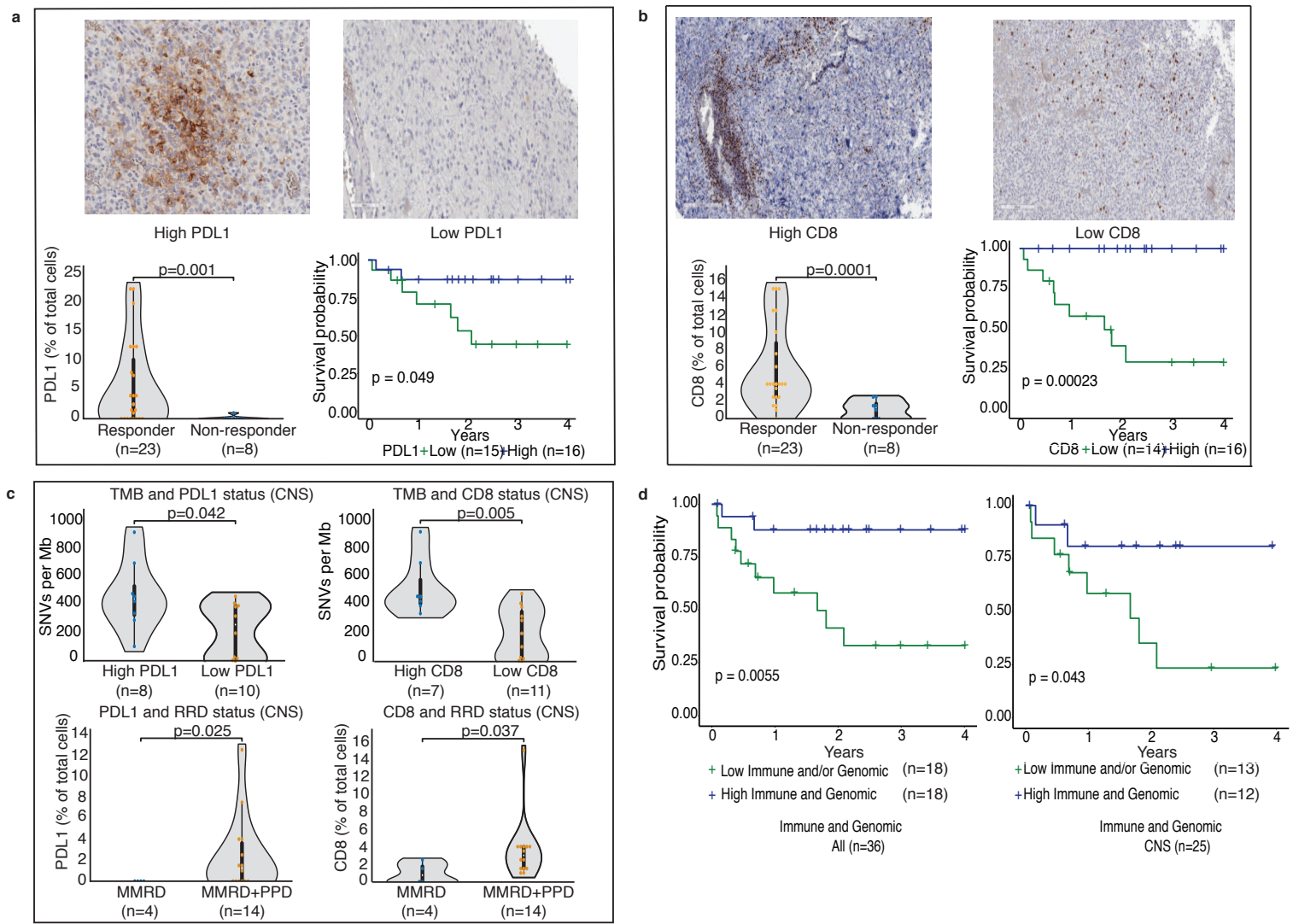

908 Fig. 5. Tumour immune microenvironment, survival and response to PD-1 blockade. (a)

909 PD-L1 expression, response and survival in all RRD cancers. Cut-off is $\geq 1 \%$ of cells

910 (Methods). (b) CD8 expression, response and survival for RRD cancers. Cut-off is $\geq 3 \%$ of

911 cells (higher than the median; Methods). For both (a) and (b), the histology depicts

912 glioblastoma at 20X magnification. (c) Association of immune markers with SNV and RRD

913 status. (d) Combined immune (PD-L1 and CD8 expression) and genomic (TMB and MS-

914 indels) and overall survival in RRD cancers. 
a

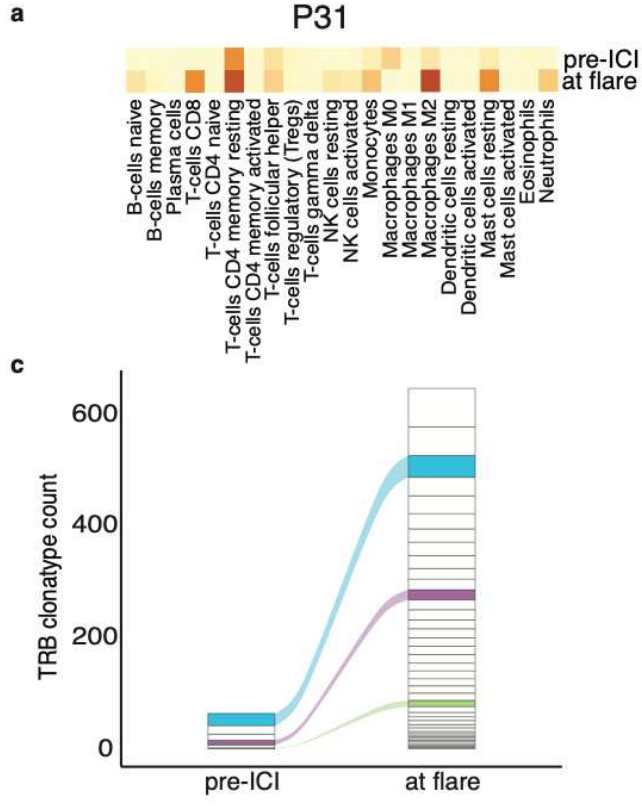

e

CD8

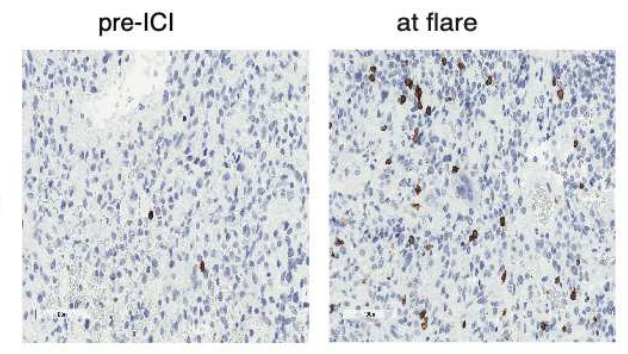

b
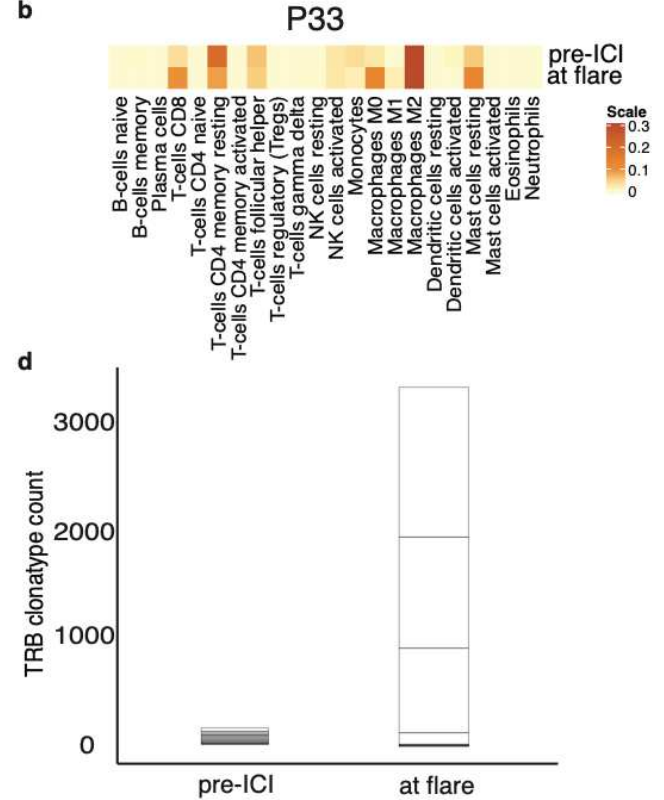

$\mathbf{f}$

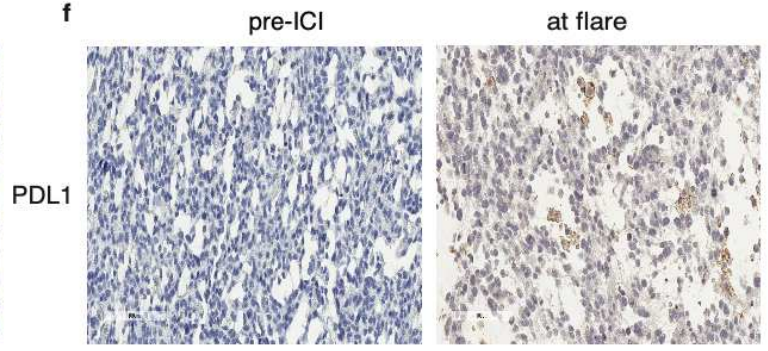

g
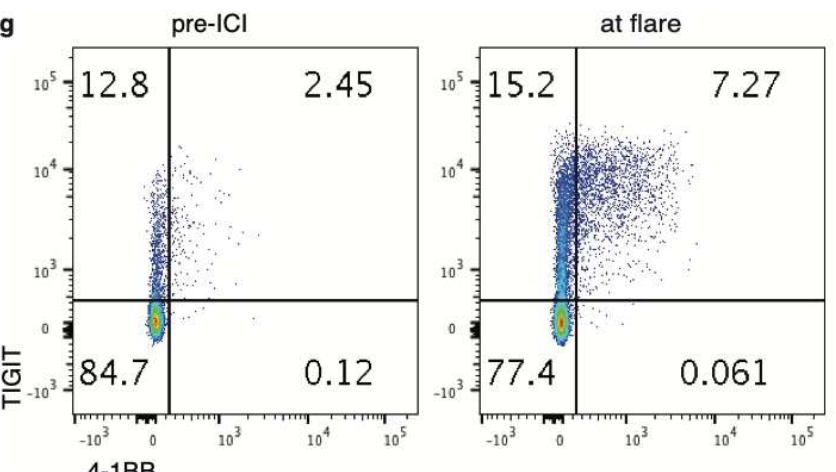

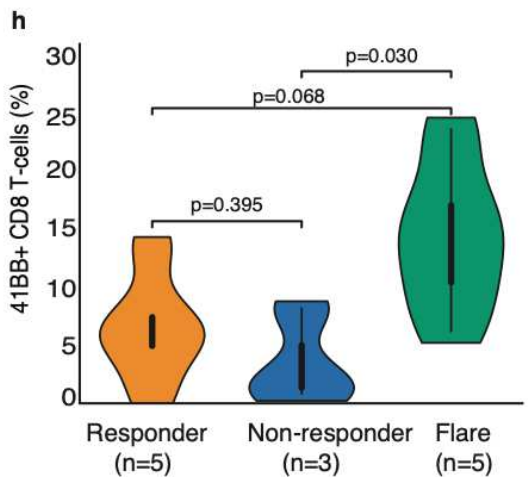

916 Fig.6. Characterization of the tumour flare response. (a-d) Analysis of 2 patients who had

917 tumour-debulking prior to therapy and at the time of flare. (a, b) Total immune cell content in

918 pre-therapy and at flare. (c, d) The corresponding CapTCR-sequencing and T-cell receptor

919 clonotype analysis in these samples. (e, f) Immunohistochemistry for PD-L1 expression, and

920 CD8-T-cell infiltration in the pre-therapy sample and at flare, as shown in the representative

$92120 \mathrm{X}$ images from the tumour sample in patient-1 (P33). (g) Representative flow cytometry plot

922 showing activation of CD+ T-cell (TIGIT and 4-1BB) from the blood sample of a patient before

923 treatment initiation and at flare. (h) $41 \mathrm{BB}+\mathrm{CD} 8+\mathrm{T}$-cells in blood from responders without

924 flare, non-responders and flare. 
a

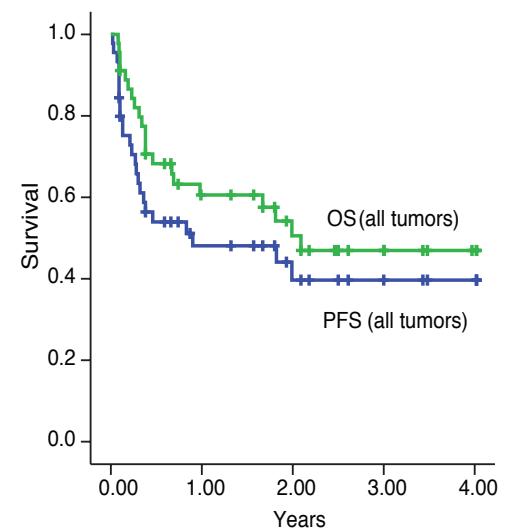

c

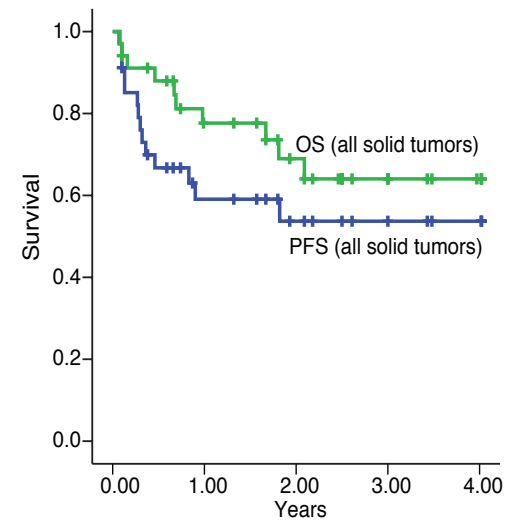

b
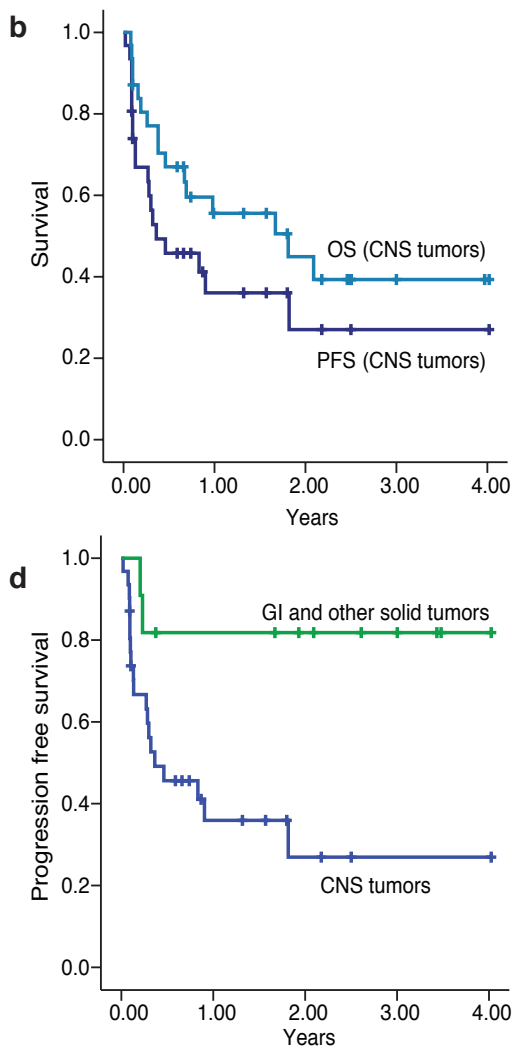

927 Extended Data Fig.1. KM estimates of progression-free and overall survival for (a) all 928 tumours, (b) CNS tumours, (c) all solid tumours (CNS and non-CNS). (d) Progression free 929 survival for non-CNS solid tumours versus CNS tumours $(\mathrm{p}=0.01)$. 

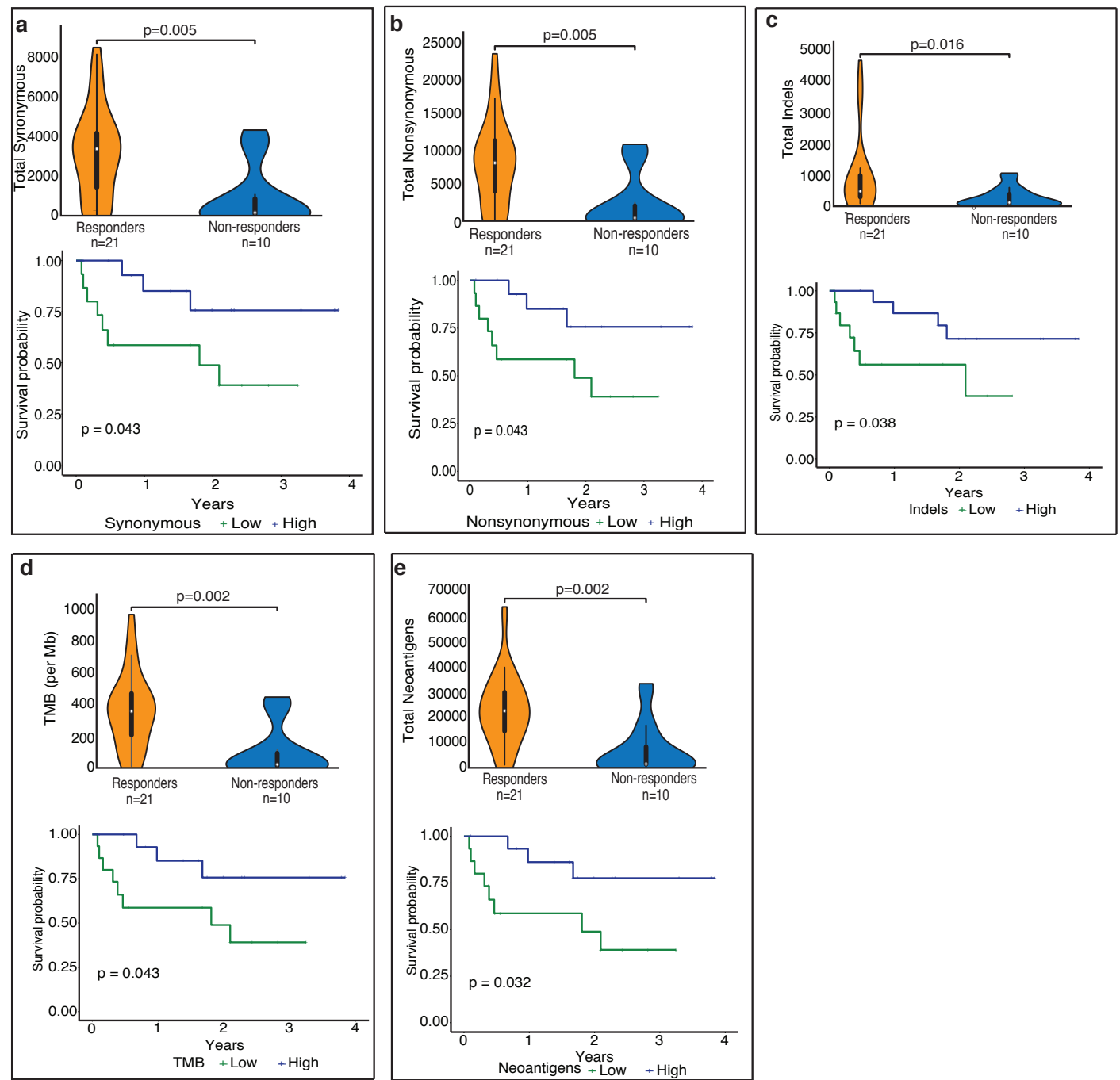

931 Extended Data Fig.2: Genomic biomarkers, survival and response to PD-1 blockade.

932 Response and overall survival by (a) total synonymous variants, (b) non-synonymous variants,

933 (c) total indels, (d) total mutations/ $\mathrm{Mb}$, and (e) neoantigens. 

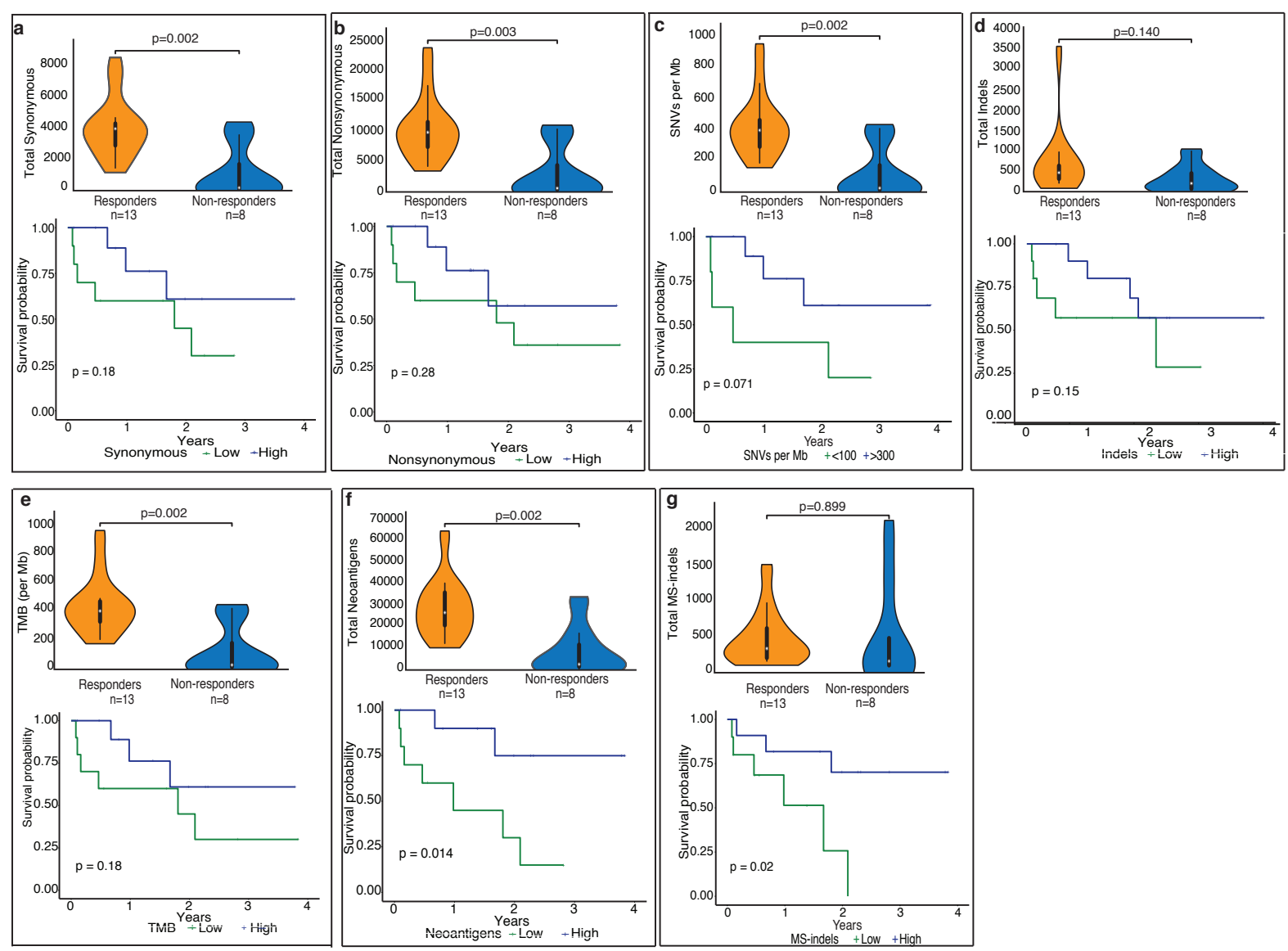

934

935 Extended Data Fig.3: CNS tumours: Genomic biomarkers, survival and response to PD-

9361 blockade. Response and overall survival for CNS tumours by (a) synonymous variants, (b)

937 non-synonymous variants, (c) SNVs/Mb, (d) total indels, (e) total mutations/ $\mathrm{Mb}$, (f)

938 neoantigens, and (g) MS-indels. 

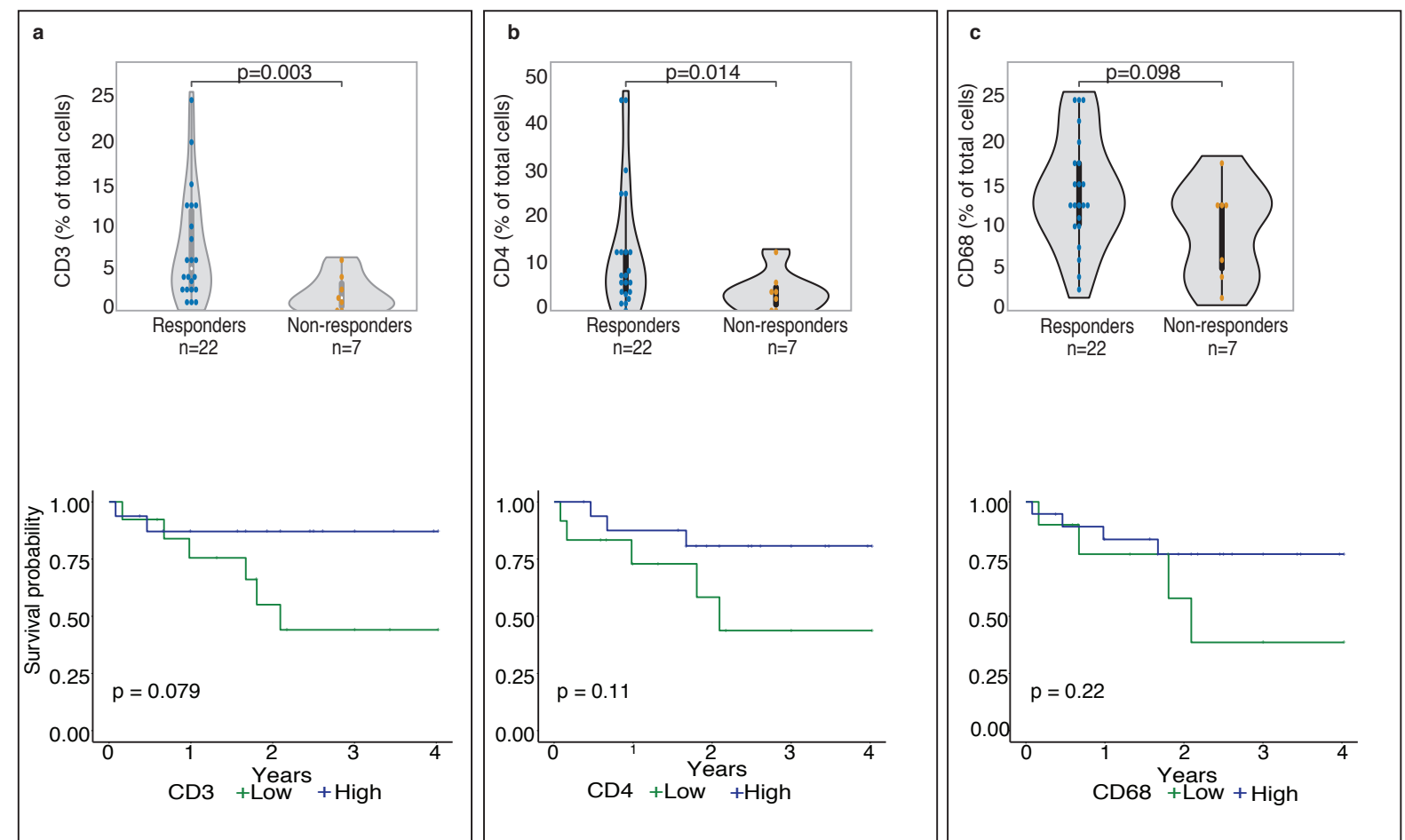

939

940 Extended Data Fig.4: Tumour immune microenvironment, survival and response to PD-

9411 blockade. Response and overall survival by (a) CD3, (b) CD4, and (c) CD8-positive T-cell 942 infiltration in the pre-ICI tumour specimens. 
a

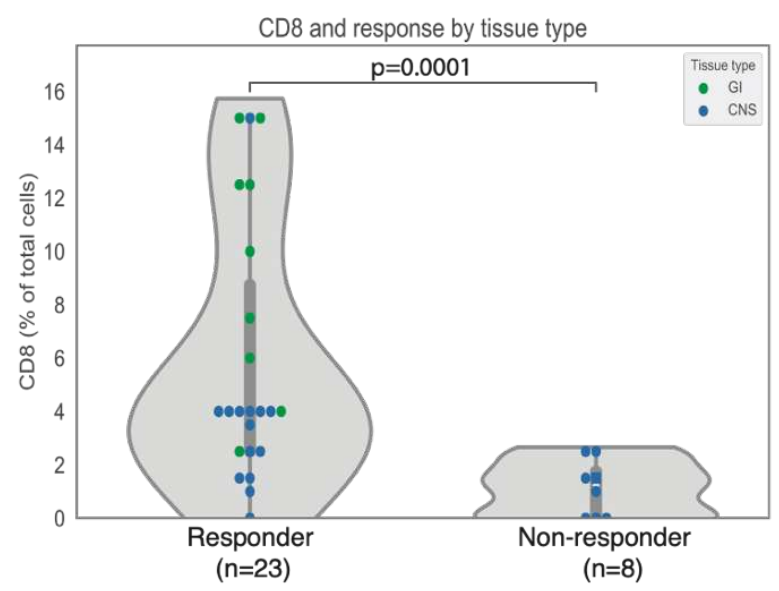

b

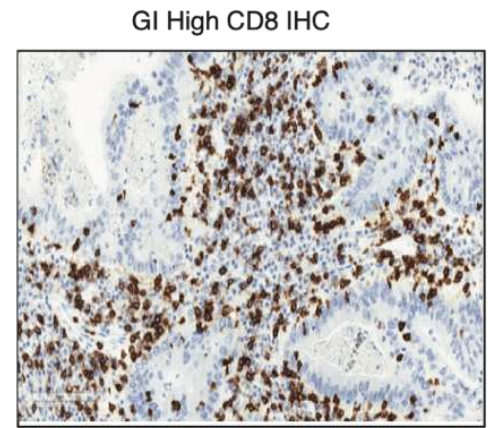

c

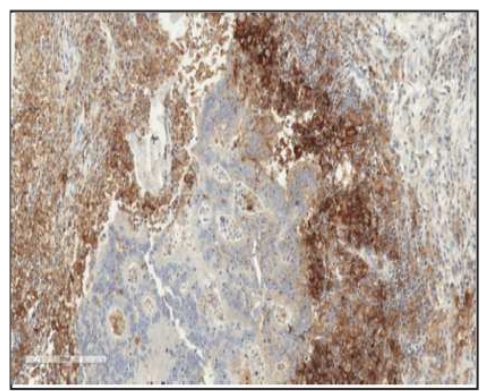

GI High PDL1 IHC

944 Extended Data Fig.5: Total CD8 cells and response to PD-1 blockade. (a) Responders had 945 higher levels of CD8 T-cell infiltration as compared to non-responders in all tumours. Green946 gastrointestinal, blue- CNS tumours. (b) Representative images from non-CNS solid tumours, 947 all of which demonstrated high CD8 and (c) PD-L1 expression. 

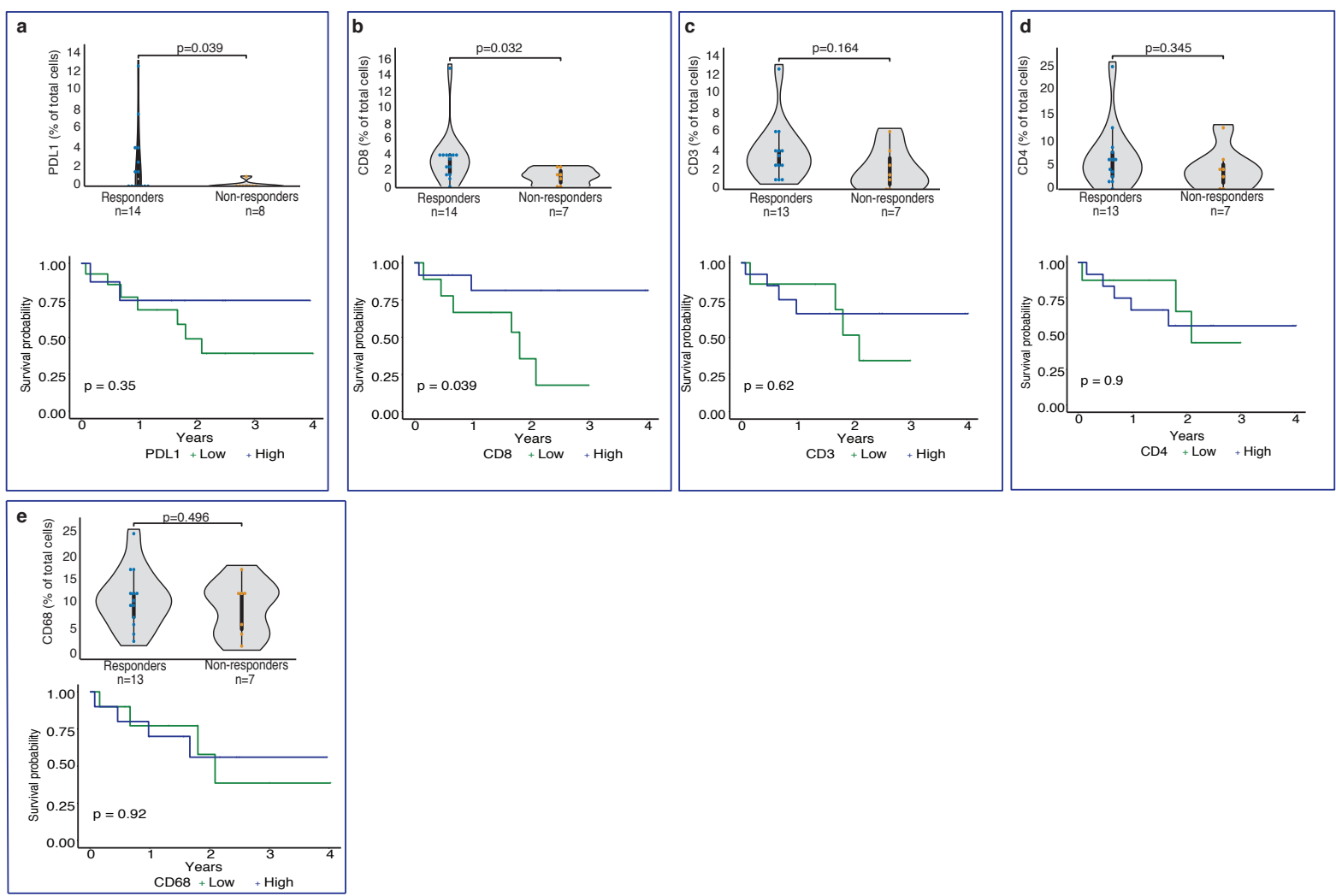

949 Extended Data Fig.6: CNS tumours: Immune microenvironment, survival and response

950 to PD-1 blockade. Response and overall survival in CNS tumours by (a) PD-L1, (b) CD8, (c) 951 CD3, (d) CD4, and (e) CD68 expression. 


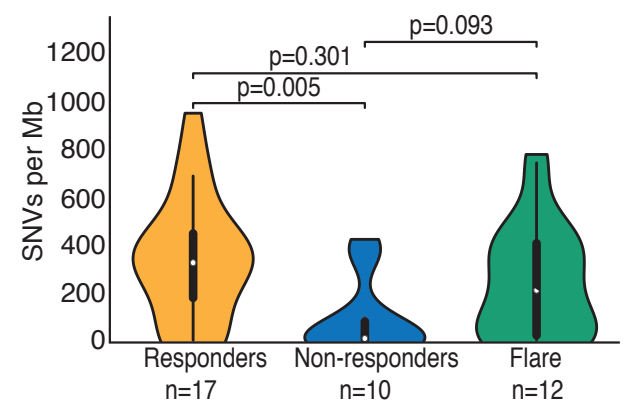

C

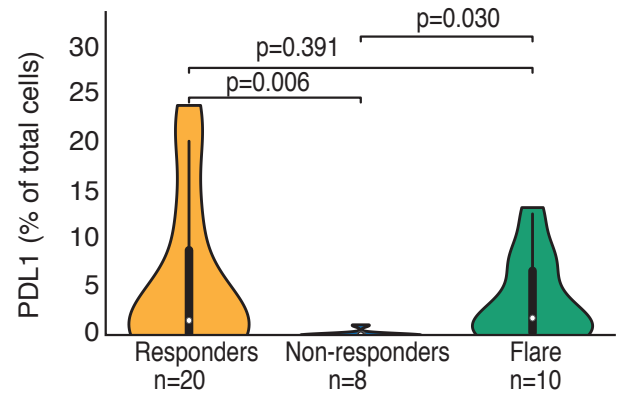

b

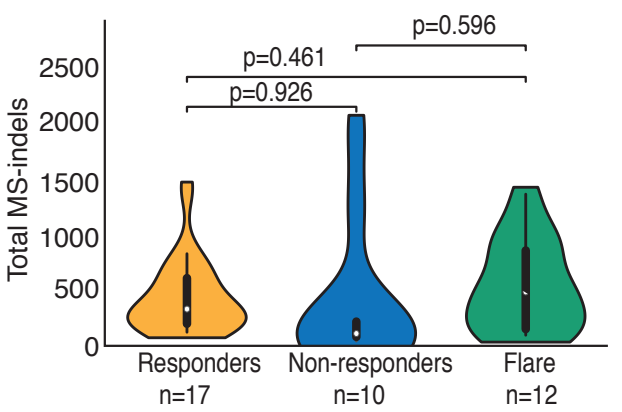

d

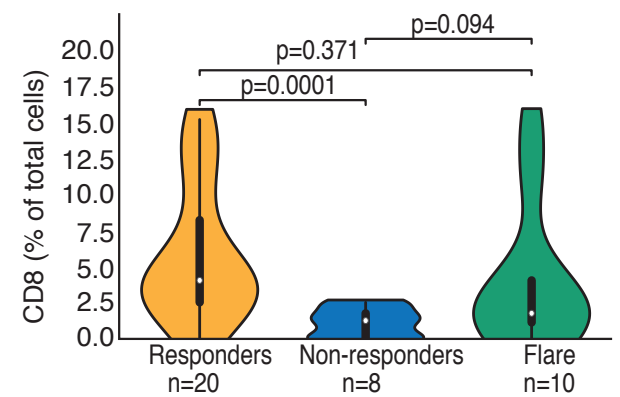

953 Extended Data Fig.7: Genomic and immune markers of response in tumours exhibiting

954 flare. Responders without flare, non-responders and flare stratified by (a) SNVs/mb, (b) MS955 indels, (c) PD-L1 and (d) CD8 expression. 
a

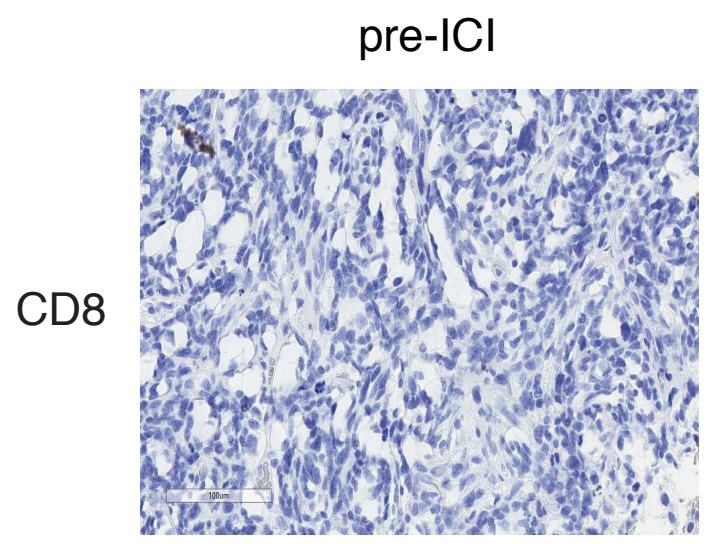

b

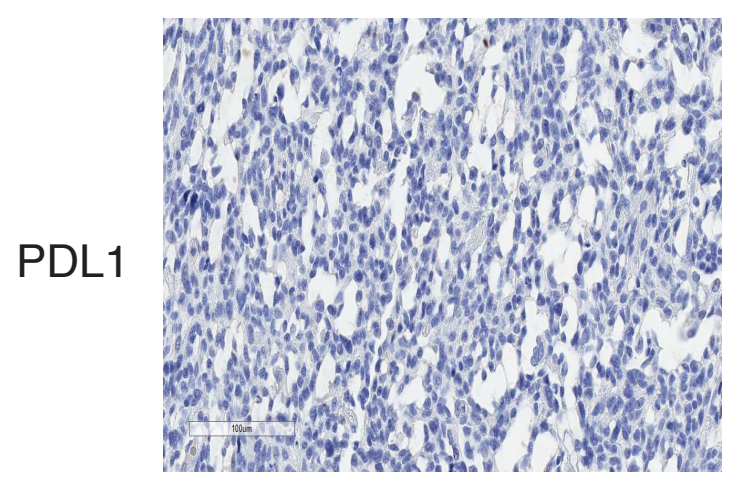

at flare
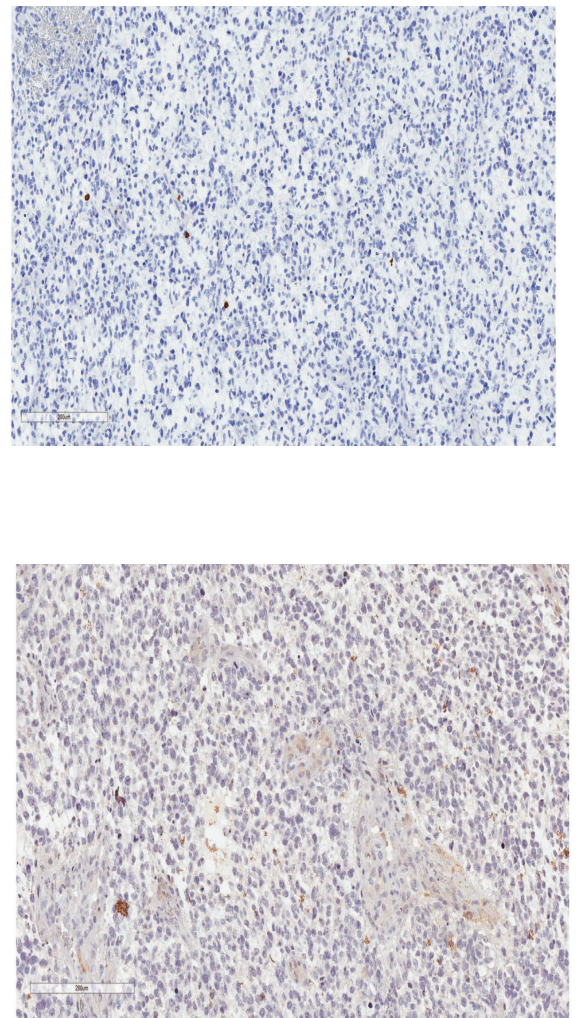

957

958 Extended Data Fig.8: Representative images (20X) from patient P31 demonstrating (a) CD8 959 and (b) PD-L1 expression in pre-ICI and flare samples. 
a

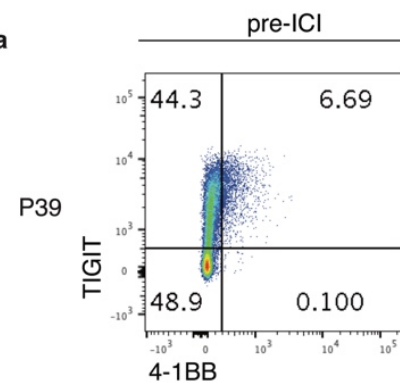

b
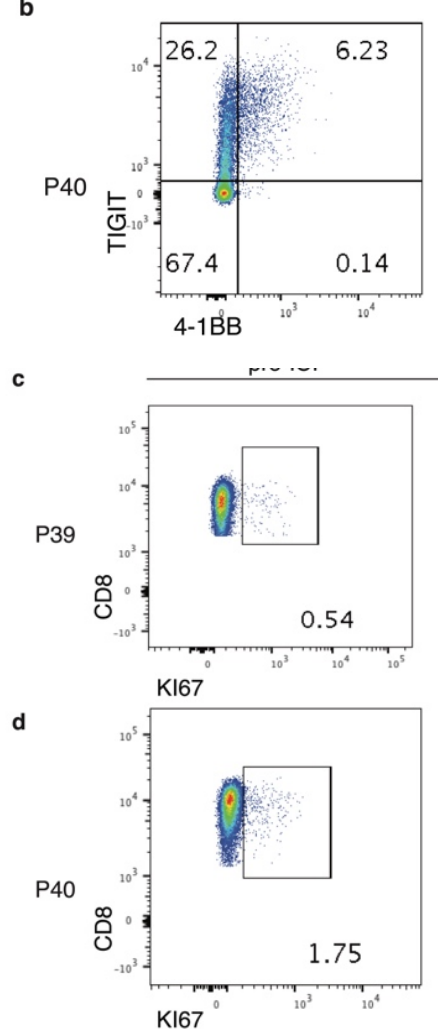

at flare
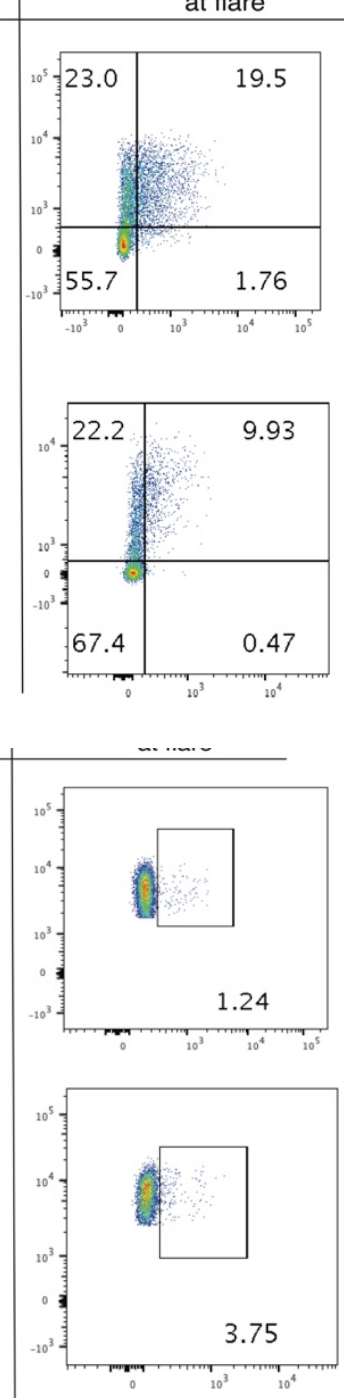

e

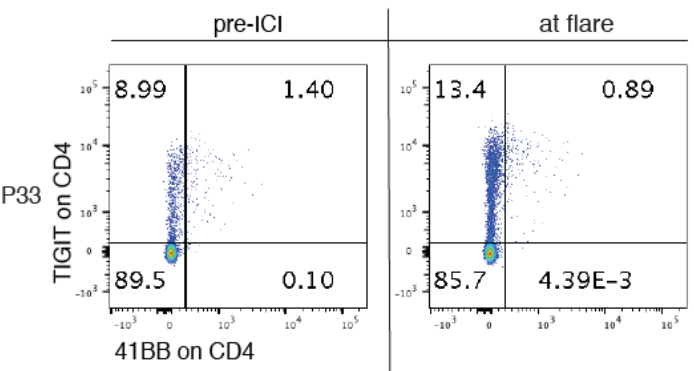

f
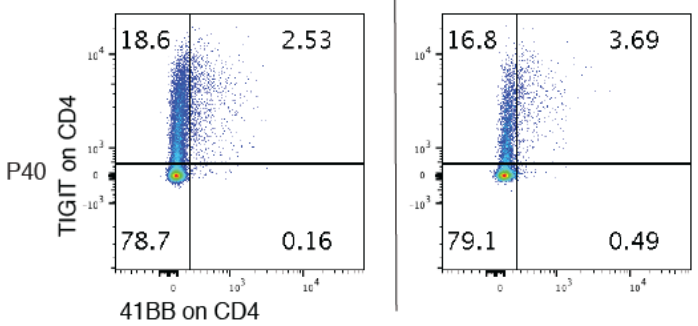

973

974

975 Extended Data Fig.9: Immune activation in patients experiencing tumour flare. Flow

976 cytometry dot-plots from two additional patients (P39, P40) comparing pre-ICI and flare

977 samples, showing (a, b) TIGIT and 4-1BB expressing CD8+ T-cells, (c, d) Ki67 expressing 978 CD8+ T-cells, and (e ,f) TIGIT and 4-1BB expressing CD4+ T-cells. 
Extended Data Table S2:

Binary logistic regression analysis for response to ICI in RRD cancers

\begin{tabular}{|c|c|c|c|}
\hline Variables & Odds ratio & $\mathbf{9 5 \%}$ CI & p-value \\
\hline Age & 1.19 & $0.8,1.6$ & 0.26 \\
\hline Male vs female gender & 0.14 & $0,6.1$ & 0.30 \\
\hline CNS vs non-CNS solid tumours & 1.15 & $0.0,-$ & 0.99 \\
\hline Nivolumab vs Pembrolizumab & 0.01 & $0,3.6$ & 0.12 \\
\hline High SNV/Mb (>median) & 0.03 & $0,0.5$ & $\underline{0.01}$ \\
\hline High MS-indel (>median) & 0.08 & $0,2.1$ & 0.13 \\
\hline
\end{tabular}

981

982

Extended Data Table S3:

983

Cox regression analysis for OS following ICI in patients with RRD cancers

\begin{tabular}{|c|c|c|c|}
\hline Variables & Odds ratio & $\mathbf{9 5 \%}$ CI & p-value \\
\hline Age & 0.88 & $0.7,1.1$ & 0.33 \\
\hline Male vs female gender & 1.20 & $0.2,6.1$ & 0.83 \\
\hline CNS vs non-CNS solid tumours & 0 & $0.0,-$ & 0.96 \\
\hline Nivolumab vs Pembrolizumab & 1.20 & $0.1,19.8$ & 0.89 \\
\hline High SNV/Mb (>median) & 5.57 & $0.9,32.4$ & $\underline{0.05}$ \\
\hline High MS-indel (>median) & 3.15 & $0.6,16.4$ & 0.17 \\
\hline
\end{tabular}

984 


\section{Figures}

a

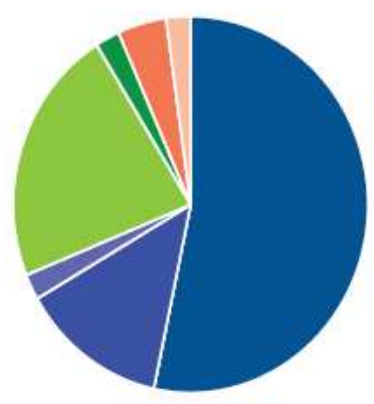

Glioma Grade IV (WHO) $(\mathrm{n}=24)$

Glioma Grade III (WHO) $(n=6)$

CNS Embryonal tumor $(n=1)$

$\square$ Gastrointestinal Cancers $(n=10)$

Urothelial Cancer $(n=1)$

T-cell Leukemia/Lymphoma $(n=2)$

$\square$ Acute Myeloid Leukemia $(n=1)$ b

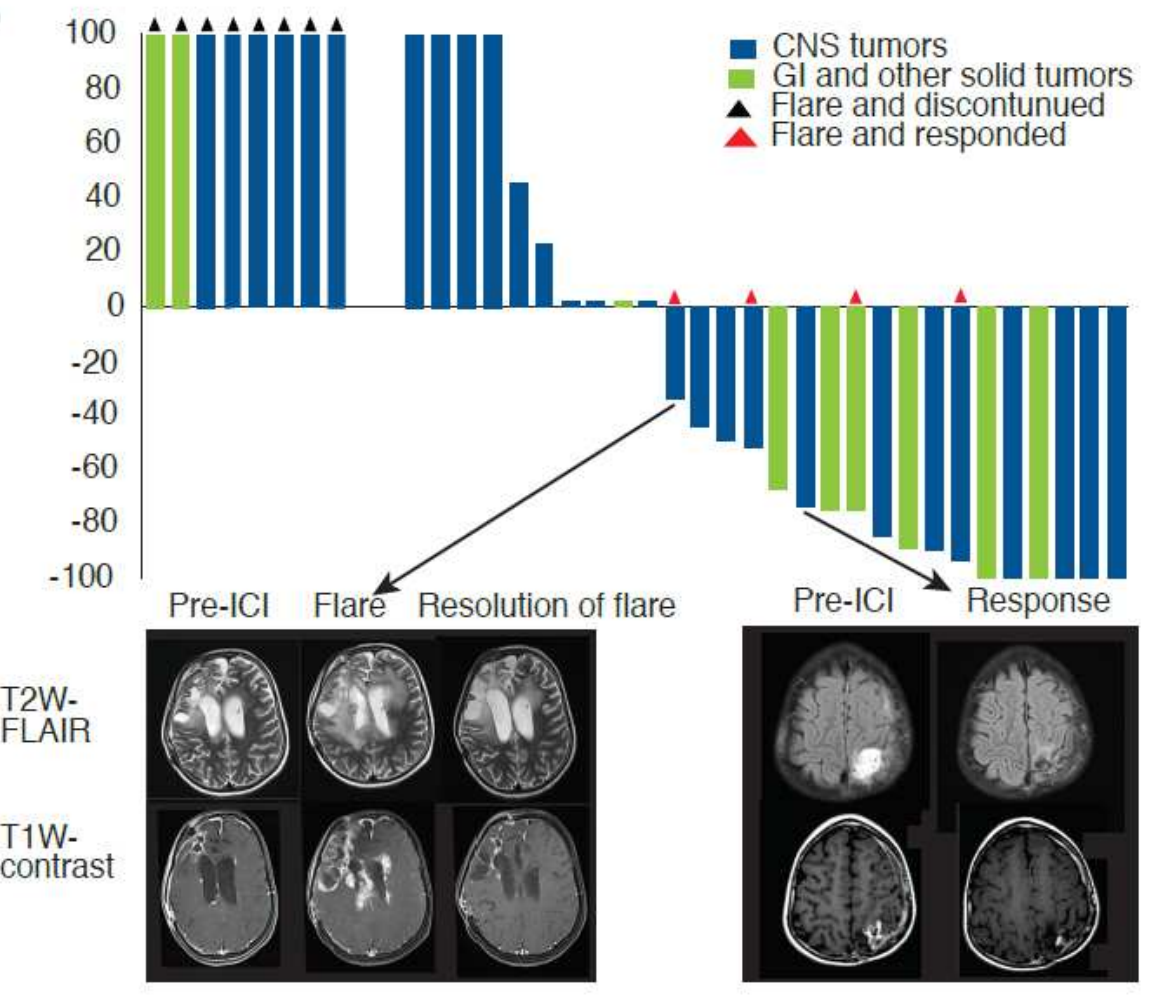

\section{Figure 1}

Clinical response to ICl across cancer types in patients with germline DNA replication repair deficiency. (a) Distribution of tumour types across 38 patients who developed 45 tumours. (b) Waterfall plot of all radiological responses in non-haematological malignancies. Values show the best fractional change in the 2 dimensions from baseline measurements as per RANO and RECIST criteria. Arrows point to representative T2-weighted FLAIR and T1-weighted contrast-enhanced MRI sequences in two patients showing flare and partial responses. 

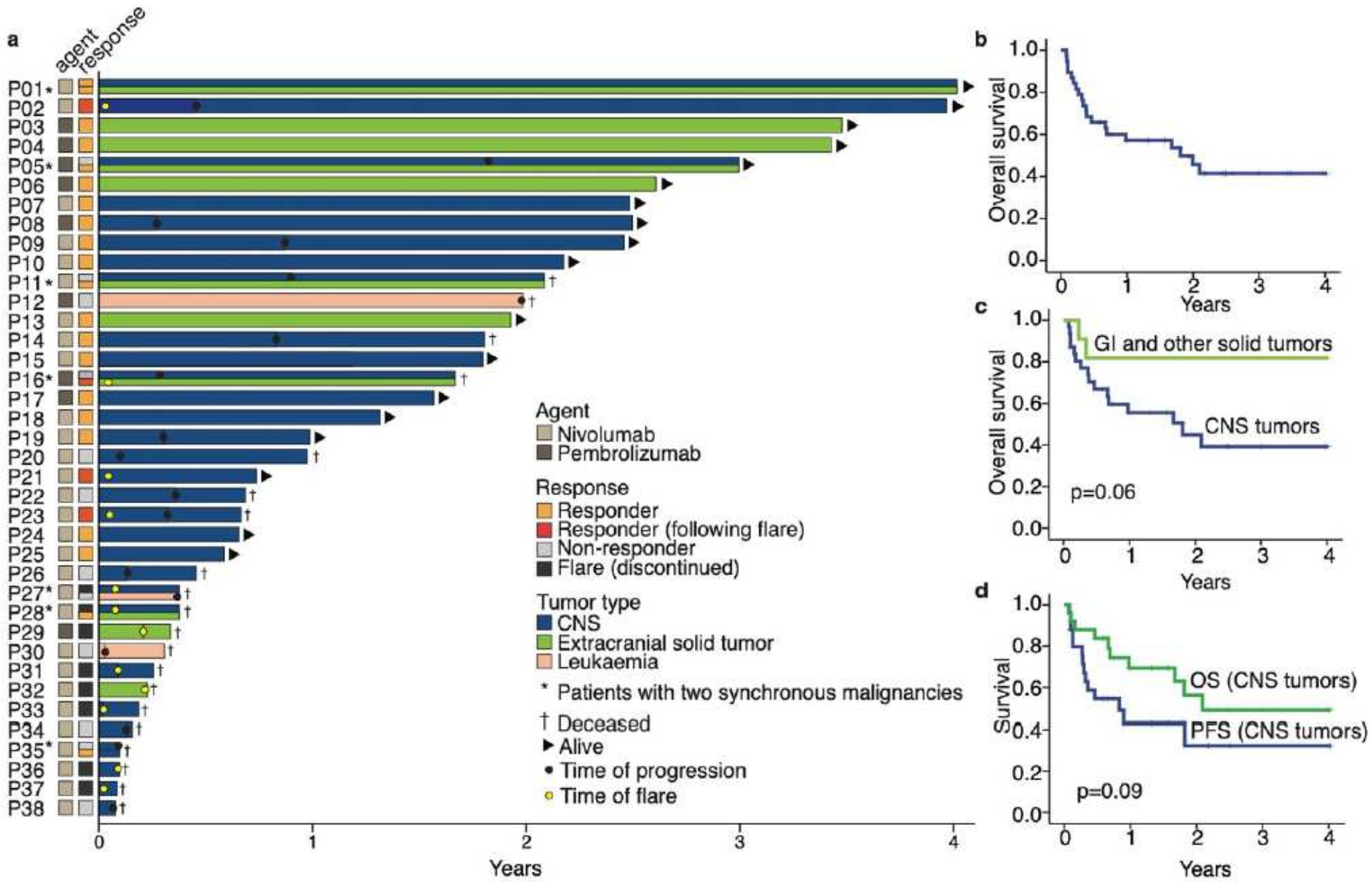

\section{Figure 2}

Patient outcome and survival by tumour type. (a) Swimmer plot by patient and tumour type. (b) KaplanMeier (KM) estimates of overall survival for all patients (c) KM estimates of overall survival as per tumour type. Median survival for CNS tumours was 21.6 months. Median survival was not reached for non-CNS solid tumours. (d) KM estimates of progression free and overall survival for CNS tumours continuing ICI therapy. Note: prolonged median survival at 24 months (estimated 3-year OS $=49.1 \%$ ) despite initial radiological progression at a median of 9.9 months (estimated 3-year $\mathrm{PFS}=32 \%$ ). 

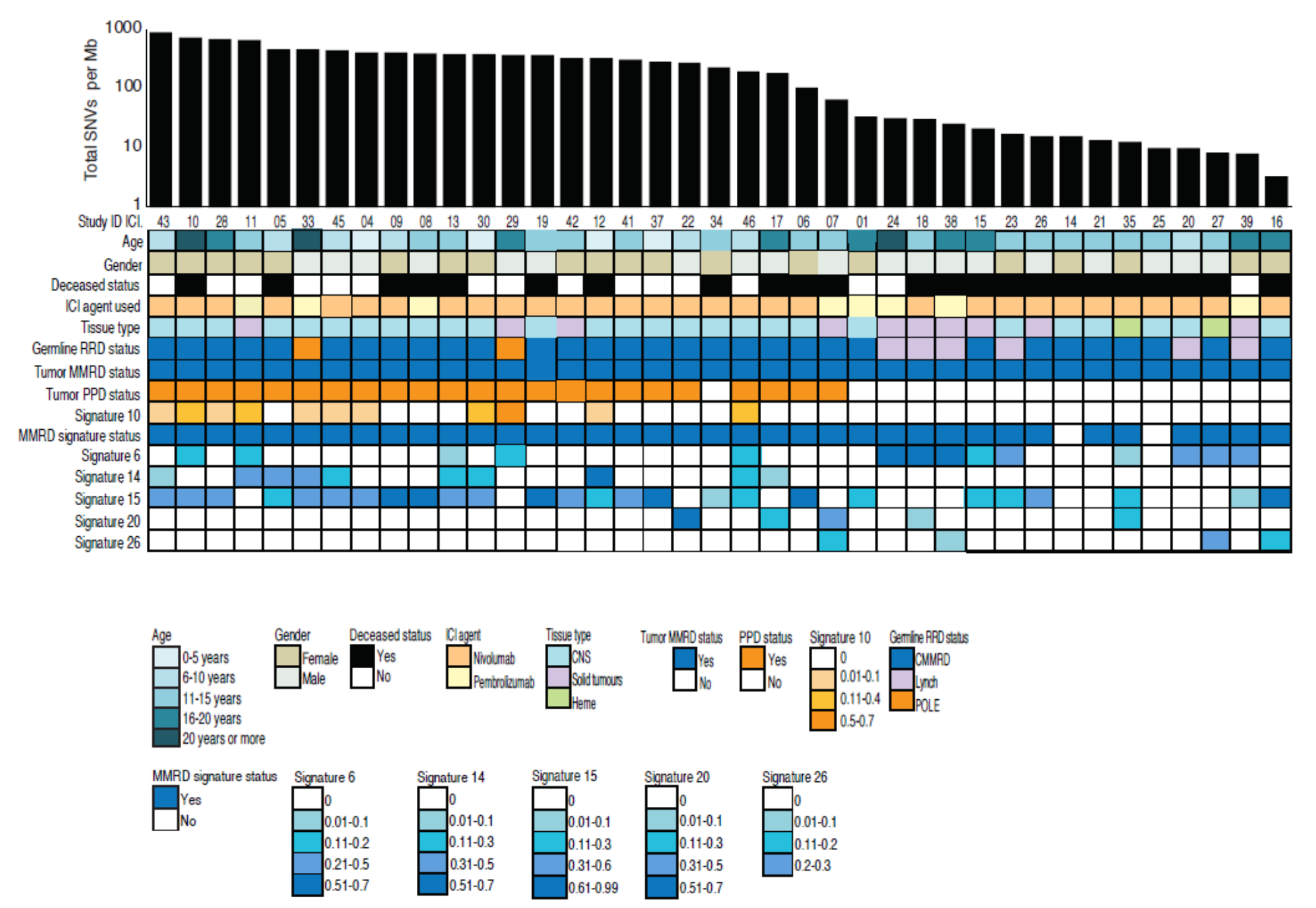

Figure 3

Genomic biomarkers, survival and response to PD1 blockade. (a) Onco-plot summarising the genomic features from 39 available paired tumour and germline exomes, and their clinical correlates. (b) Response and overall survival (OS) by single nucleotide variants (SNVs) per Mb. For survival analysis, median SNV burden was used. (c) SNVs as a function of MMRD (blue) and MMRD+PPD (orange) status (left), and response association with both SNV and RRD status. (c) Response and overall survival (OS) by microsatellite indels (MS indels). For survival analysis, median MS-indel values were used. (d) Response and overall survival by total MS-indel count for MMRD and MMRD+PPD cancers separately. (e) KaplanMeier (KM) estimates using combined SNVs/Mb and MS-indel in all RRD cancers. (Abbreviations: MMRD: mismatch-repair deficiency; PPD: Polymerase-proofreading deficiency; MS-indel: microsatellite insertion/ deletion.) 

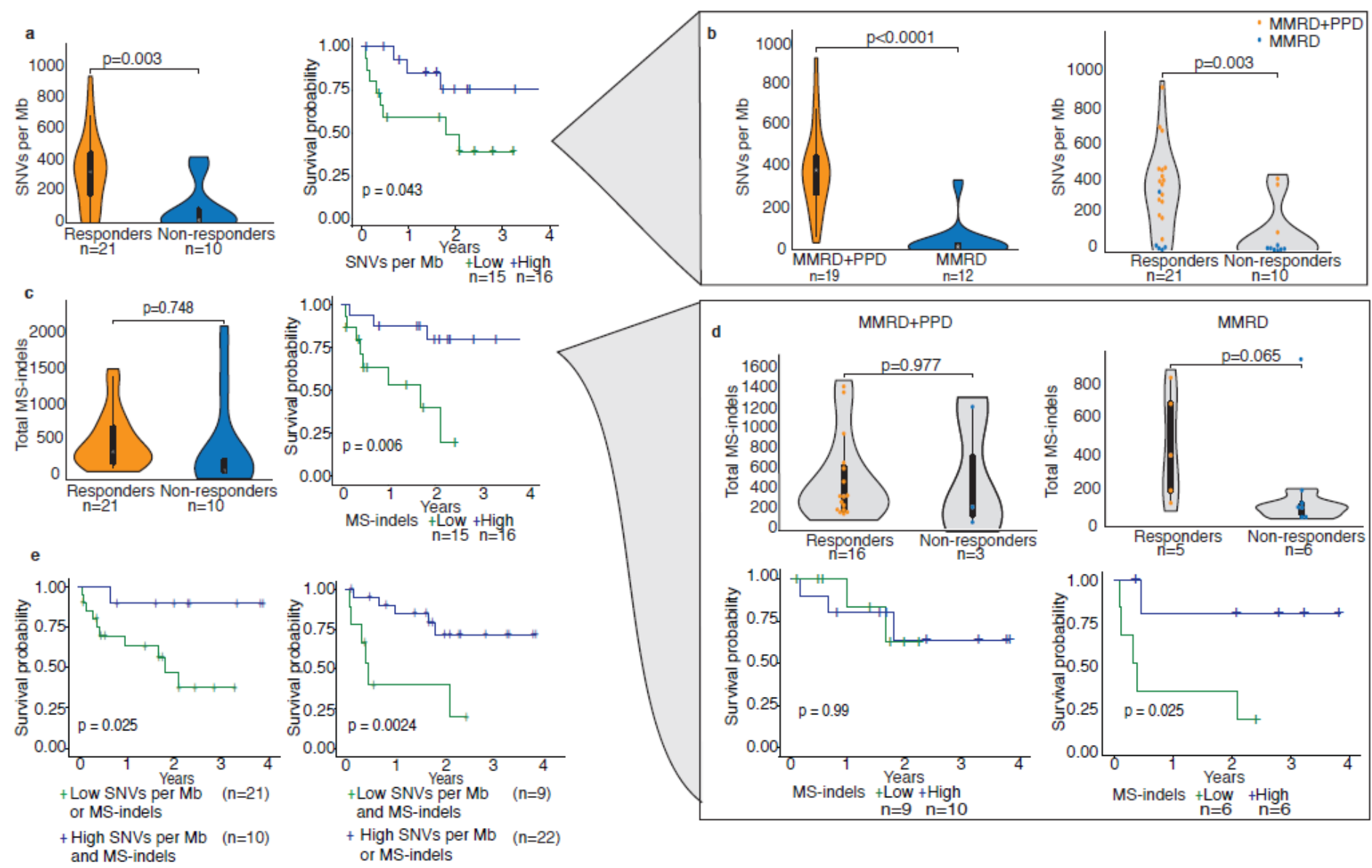

\section{Figure 4}

Genomic biomarkers, survival and response to PD1 blockade. (a) Onco-plot summarising the genomic features from 39 available paired tumour and germline exomes, and their clinical correlates. (b) Response and overall survival (OS) by single nucleotide variants (SNVs) per Mb. For survival analysis, median SNV burden was used. (c) SNVs as a function of MMRD (blue) and MMRD+PPD (orange) status (left), and response association with both SNV and RRD status. (c) Response and overall survival (OS) by microsatellite indels (MS indels). For survival analysis, median MS-indel values were used. (d) Response and overall survival by total MS-indel count for MMRD and MMRD+PPD cancers separately. (e) KaplanMeier (KM) estimates using combined SNVs/Mb and MS-indel in all RRD cancers. (Abbreviations: MMRD: mismatch-repair deficiency; PPD: Polymerase-proofreading deficiency; MS-indel: microsatellite insertion/ deletion.) 

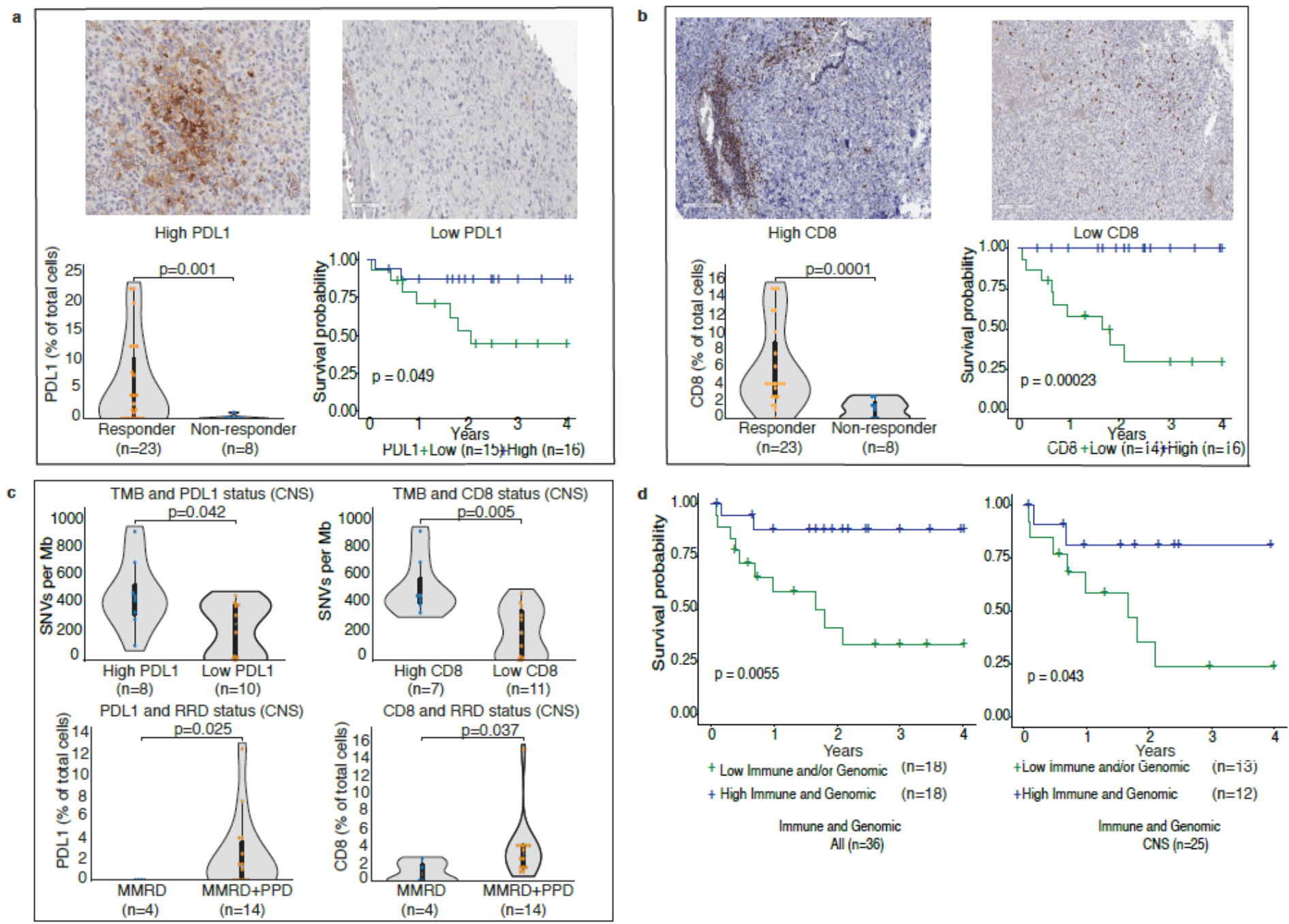

\section{Figure 5}

Tumour immune microenvironment, survival and response to PD-1 blockade. (a) PD-L1 expression, response and survival in all RRD cancers. Cut-off is $\geq 1 \%$ of cells (Methods). (b) CD8 expression, response and survival for RRD cancers. Cut-off is $\geq 3 \%$ of cells (higher than the median; Methods). For both (a) and (b), the histology depicts glioblastoma at 20X magnification. (c) Association of immune markers with SNV and RRD status. (d) Combined immune (PD-L1 and CD8 expression) and genomic (TMB and MS indels) and overall survival in RRD cancers. 
a

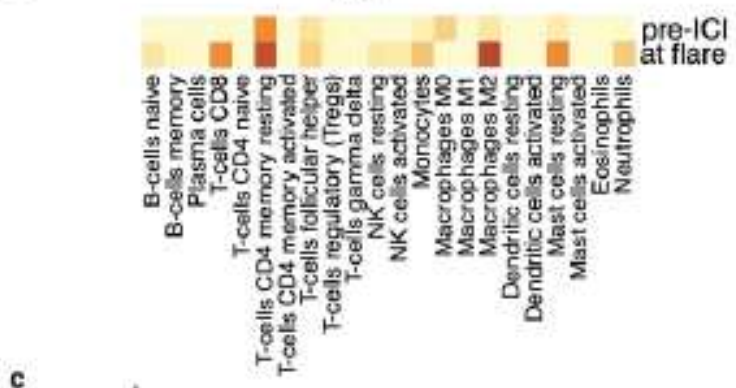

c

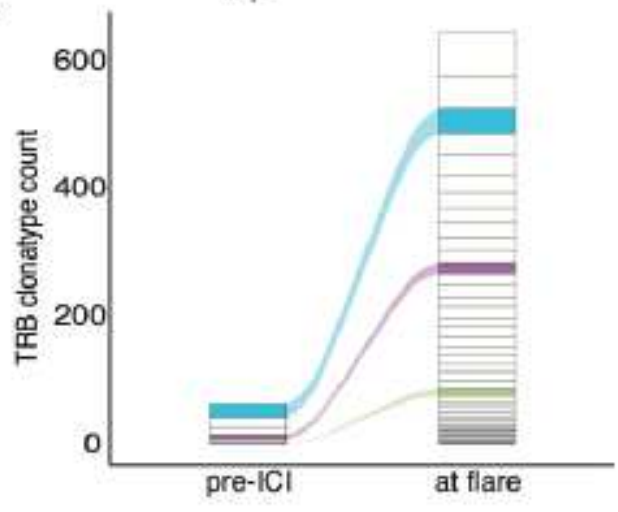

e

pre-ICI

$\operatorname{CDB}$

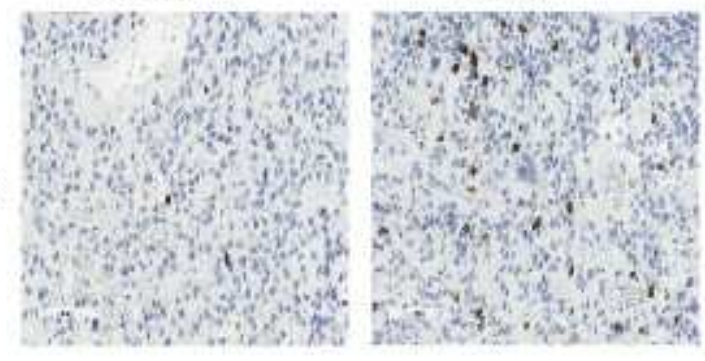

b
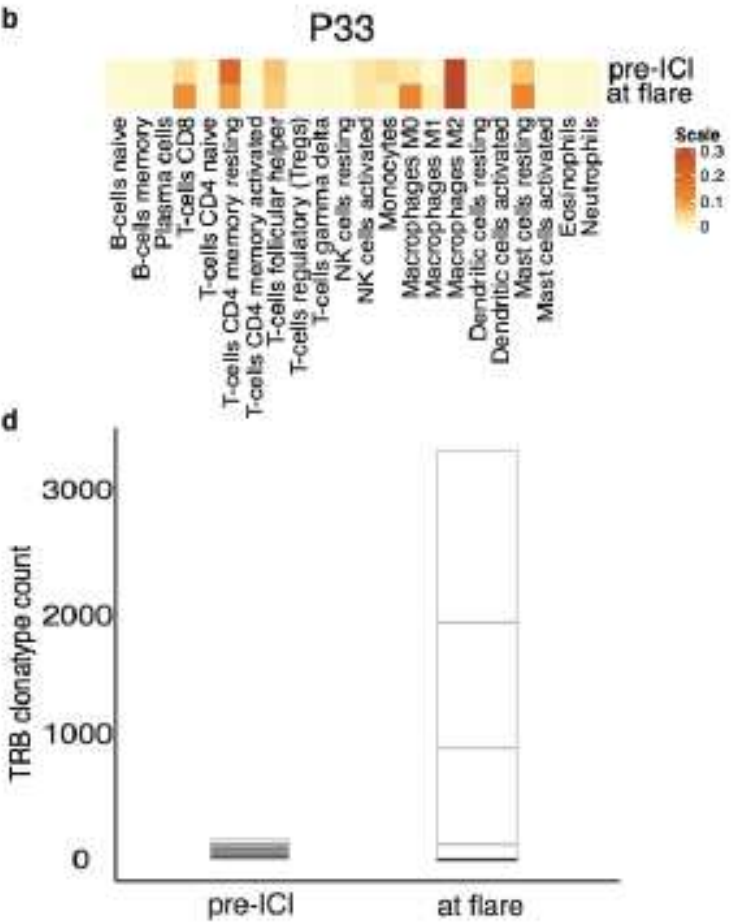

1

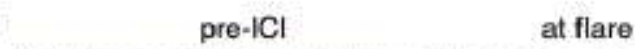

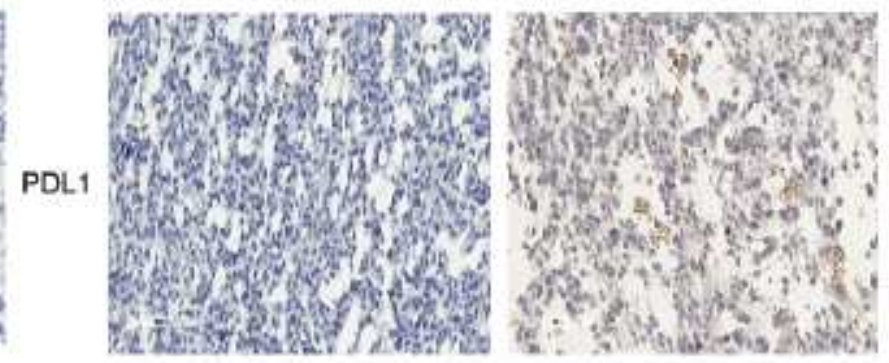
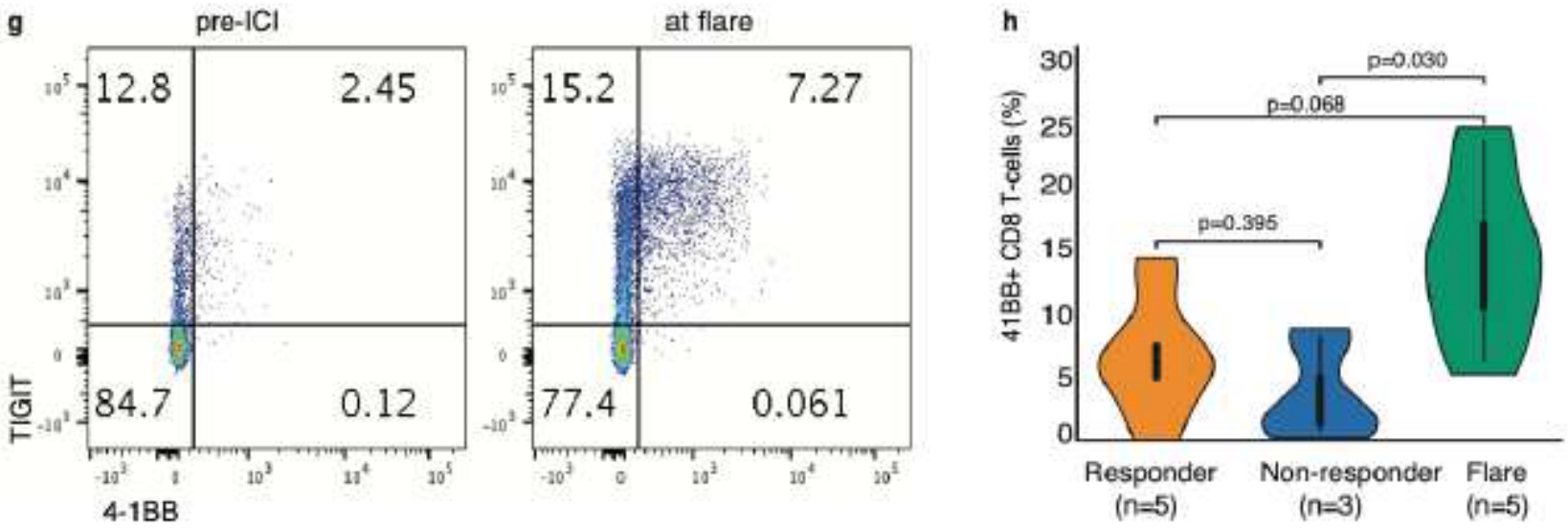

Figure 6

Characterization of the tumour flare response. (a-d) Analysis of 2 patients who had tumour-debulking prior to therapy and at the time of flare. $(a, b)$ Total immune cell content in pre-therapy and at flare. (c, d) The corresponding CapTCR-sequencing and T-cell receptor clonotype analysis in these samples. $(e, f)$ Immunohistochemistry for PD-L1 expression, and CD8-T-cell infiltration in the pre-therapy sample and at flare, as shown in the representative 20X images from the tumour sample in patient-1 (P33). (g) 
Representative flow cytometry plot showing activation of CD+ T-cell (TIGIT and 4-1BB) from the blood sample of a patient before treatment initiation and at flare. (h) 41BB+ CD8+ T-cells in blood from responders without flare, non-responders and flare.

\section{Supplementary Files}

This is a list of supplementary files associated with this preprint. Click to download.

- ExtendedDataTableS1FINAL.pdf 